EPJ manuscript No.

(will be inserted by the editor)

\title{
Heavy nuclei: introduction to Density Functional Theory and variations on the theme
}

\author{
Gianluca Colò ${ }^{1}$ \\ 1 Dipartimento di Fisica, Università degli Studi di Milano \\ 2 INFN, Sezione di Milano, Via Celoria 16, 20133 Milano (Italy)
}

Received: date / Revised version: date

\begin{abstract}
Density Functional Theory (DFT) is based on the rigorous Hohenberg-Kohn (HK) theorem, that has been first introduced for electronic systems but holds for all fermionic systems including atomic nuclei. Currently, DFT has reached a high level of sophistication, and represents a suitable tool for a self-consistent, quantitative description of bulk nuclear properties (like masses, radii and deformations) of all nuclei except, perhaps, the lightest ones. Furthermore, time-dependent calculations within the same framework account for the observed properties of several different types of nuclear collective motion. In this lecture, we introduce these topics by assuming only a previous knowledge of general quantum mechanics and of basic nuclear phenomenology (we actually review some parts of it).
\end{abstract}

PACS. PACS-key discribing text of that key - PACS-key discribing text of that key

\section{Introduction}

Nuclear physics is often believed to be a complex subject. Perhaps, one of the reasons consists in the huge variety of questions that one may raise, together with the difficulties in answering them. Nuclei are quantum many-body systems [1], and it is well known that these are intractable with simple theoretical methods when the number of components is neither very small nor very large (in nuclei we have $\approx 10^{0}-10^{2}$ particles). This statement holds even for many-body systems where the basic interaction is known, like in the Coulomb case: in fact, even the ensembles of ions and electrons like molecules, liquids or solids, display a very rich phenomenology (either weak or strong correlations, emergent properties, phase transitions etc.), that is far from being satisfactorily understood. This is even more true for nuclei, because the strong force in the medium is much less known than the Coulomb force. We do not have at our disposal, so far, a unique and neat way to describe the interaction between nucleons, at the scale which is relevant for nuclear systems, by starting from the underlying Quantum Chromo Dynamics (QCD). Recent progress are being made, though [2-4].

It is better to define the goals of this lecture at the very beginning. The goal here is reaching an overall understanding of nuclear structure based on a microscopic modelling that can be applied to most of the nuclei, and to their overall features such as ground-state properties (mass, shape, normal vs. superfluid character) or collective excitations. As said above, the so-called ab initio models, partly discussed in this volume, are experiencing significant progresses, but they are not yet a quantitative tool to systematically predict the properties of both ground and excited states, throughout the whole isotope chart. DFT has, instead, this feature. It is based on the HK theorem and is, as such, a microscopic theory. Within its framework, with about 10 free parameters and a remarkable conceptual simplicity, one can reproduce reasonably well a large fraction of the observed phenomena concerning ground- and excited-states of atomic nuclei. Other models may be more accurate but have a stronger focus, namely they are usually applied to specific sub-sets of data and/or they employ more complex strategies and more parameters. Without denying that the complementarity of different models is often an asset in physics, we can safely state that DFT is a good compromise between simplicity and reproduction of experimental data. One shortcoming is that there exist several different realizations of DFT as we discuss below. There is no unique way to build a so-called energy density functional (EDF), and no obvious path for systematic improvements as it happens in theories based on perturbative expansions.

The outline of this lecture is as follows. Some elementary phenomenology of medium-heavy nuclei is reviewed in Sec. 2. The idea is to set the stage for the basic features that a sound theory, like DFT, must account for. The ideas of DFT are introduced in Sec. 3, while the EDFs in use are discussed in Sec. 4. Nuclear superfluidity is the subject 

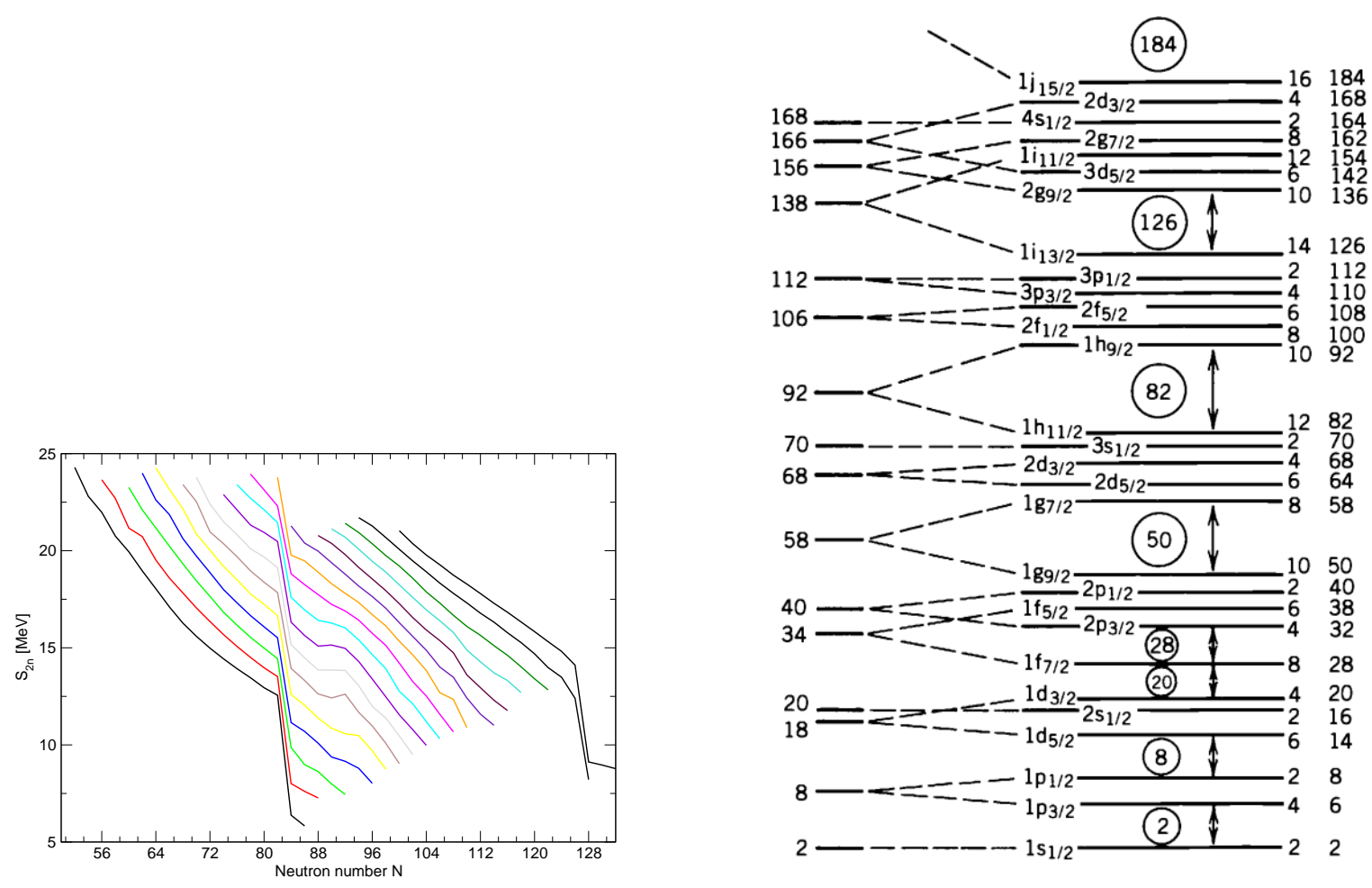

Fig. 1. (Left panel) Two-neutron separation energies $S_{2 n}$ as a function of the neutron number, for the isotopes with $50<Z<82$ (the proton number increases from the bottom left to the upper right lines). (Right panel) Shell model orbitals (cf. the text for a short discussion).

of Sec. 5. A few examples of ground-state and excited-states calculations are illustrated in Secs. 6 and 7 , respectively. Finally, some very short conclusions are drawn in Sec. 8.

Obviously, reading this paper can be seen as barely scratching the surface of the complex topics that I shall describe. While I believe that such reading is useful for freshmen in nuclear structure, quoting some references that are meant for further study is very appropriate here. There are standard textbooks that constitute a useful starting point for the study of nuclear structure [5-8]. DFT in nuclear physics has been first reviewed in [9], but the reader can also profit from Refs. [10,11]. Specific and good overviews for relativistic DFT can be found in [12,13] (I will stick mainly to the nonrelativistic version of DFT in this lecture for pedagogical reasons, since I presume that students are more familiar with the Schrödinger equation than with the Dirac equation). There are many lectures available on the web, and an ambitious and interesting attempt to introduce the subject of DFT in nuclear physics can be found on the archive [14]. Recently, I have tried to give an orienteering for students interested in nuclear DFT and in its relationships with DFT for electronic systems in the lectures given at the International School of Physics E. Fermi (Course 201, Ref. $[15])$.

\section{Review of basic phenomenology}

\subsection{Independent particle motion in nuclei: evidence for the shell structure}

The appearance of magic numbers is certainly the strongest evidence that nucleons move in the nucleus, to a first approximation, as independent particles. Nuclei having a number of protons, or neutrons, equal to 2, 8, 20, 50, 82, 126 etc., are characterised by a sudden drop when the two-neutron or two-proton separation energies $S_{2 n}$ or $S_{2 p}$ are displayed as a function of the particle number. $S_{2 n}$ is equal to $B(N, Z)-B(N-2, Z)$, where $B$ is the total binding energy (and similarly one can write for protons): in other words, when a nucleus is magic in either neutrons or protons, the energy to extract two nucleons undergoes a sudden change because an orbital shell is filled. This is evident from the left panel of Fig. 1, where the two-neutron separation energies are displayed as a function of the neutron number for the heavy isotopes that range from $\operatorname{Sn}(Z=50)$ to $\mathrm{Pb}(Z=82)$. This reminds the case of atoms, where the trend of the first ionization energies displays the evidence of the atomic shells. Note that in nuclei odd-even effects 
are pronounced [see the pairing term in the semi-empirical mass formula below, Eq. (5), and the discussion in Sec. 5], and this is why we consider two-particle separation energies.

The first magic numbers are the same for electrons in atoms and nucleons in nuclei, while starting from 50 one can see the difference that is produced by the strong nuclear spin-orbit force. In Fig. 1, the orbitals for nucleons (either neutrons or protons) in a central potential like the well-known Woods-Saxon one ${ }^{1}$ are shown on the left side while the action of the spin-orbit is visible on the right side. The number of particles for each orbit, and the cumulated number, are on the right edge of the figure.

Spectroscopy of the ground and excited states around magicity is consistent with the picture of nucleons occupying the orbitals depicted in Fig. 1. For instance, nuclei with an extra particle (or hole) with respect to a magic core have angular momentum and parity in their ground-state that are consistent with the contribution of the single extraparticle. ${ }^{17} \mathrm{O}$ has 9 neutrons and 8 protons and has a $J^{\pi}=5 / 2^{+}$ground-state, in agreement with the picture of a saturated ${ }^{16} \mathrm{O}$ plus a neutron in the $1 \mathrm{~d}_{5 / 2}$ orbital.

In keeping with the title of this volume and of the school, we should remind that a number of evidences have already been collected, pointing to the fact that the standard shell structure changes when going away from the stability valley, that is, when nuclei become neutron-rich (or neutron-deficient) [16]. In neutron-rich nuclei the proton potential becomes deeper, because of the strong neutron-proton interaction; neutrons occupy higher and higher levels, close to the continuum, that modify the shape of the average potential with respect to the case of stable nuclei, especially in light systems [17]. Thus, the idea of permanent magic numbers and also of a global mean-field should be abandoned. EDFs should be, instead, able to predict the shell changes because the average potential is an output and not a starting point, as we discuss below.

\subsection{Independent particle motion in nuclei: nucleon mean-free path}

What we have said so far, concerns nucleons that are bound inside the nucleus. If we consider an external nucleon that impinges on the nucleus, a further evidence for independent particle motion comes from the analysis of nuclear "transparency". From elementary quantum mechanics, it is well known that the elastic scattering cross section reads

$$
\frac{d \sigma}{d \Omega}=|f(\theta)|^{2}
$$

where $f$ is the scattering amplitude. In the simple case of a central potential and neglecting spin, it is also known from elementary textbooks that $f$ is related to the phase shift, namely to the shape and the values of the radial effective potential that the scattered particle has felt. The systematic analysis of low-energy neutron scattering off nuclei has produced a lot of information about such potentials. There exist local and global parameterizations (see, e.g., [18] and references therein, in particular those quoted in the caption of Table 12; see also Sec. 10.8 of [8]). In all of them, the potential has both a real, $V$, and imaginary part, $W$. The imaginary part is related to absorbtion or, more precisely, to all processes that remove the neutron from the elastic channel. From it, we can extract the neutron mean-free path.

If we assume that the potential is uniform, we can write the single-particle energy $\varepsilon$ as

$$
\varepsilon=\frac{\hbar^{2} k^{2}}{2 m}+V+i W
$$

where $m$ is the nucleon mass and $k$ is the momentum in units of $\hbar$. The previous equation can be inverted to give $k$ and, if $W$ is small with respect to $V$ so that a first-order approximation holds, we find

$$
k=\sqrt{\frac{2 m(\varepsilon-V)}{\hbar^{2}}}\left(1-\frac{i}{2} \frac{W}{\varepsilon-V}\right) \equiv k_{R}+i k_{I} .
$$

The plane wave associated with the impinging nucleon becomes

$$
e^{i k z}=e^{i k_{R} z} e^{-k_{I} z}=e^{i k_{R} z} e^{-\frac{z}{\lambda}}
$$

where the last equality defines the nucleon mean free path $\lambda=\frac{2}{k_{I}}$. The data on $W[8,18]$ suggest that at low-energy a nucleon has a mean free part that is of the order of the nuclear radius. Together with the shell structure, this is a further evidence of the independent particle motion in nuclei.

${ }^{1}$ Cf., e.g., Sec. 3.1 of Ref. [7]. 

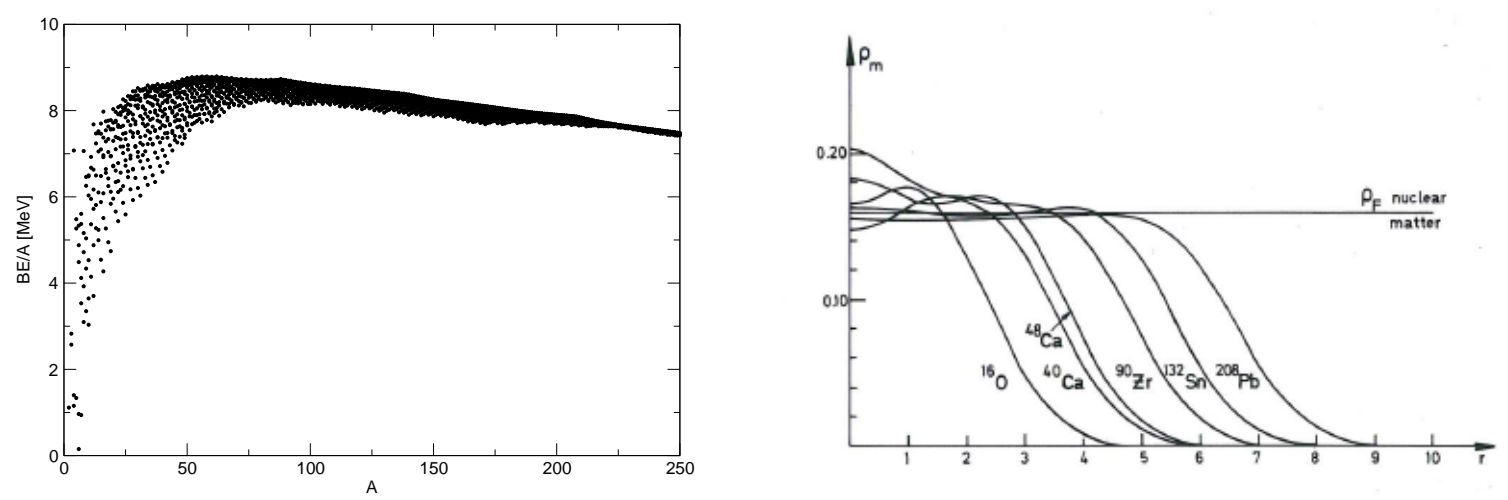

Fig. 2. (Left panel) Binding energy per particle $B / A$ as a function of the mass number $A$ (data from Ref. [19,20]). (Right panel) Nuclear matter density for few nuclei. The horizontal line marks the saturation density (figure taken from Ref. [6]).

\subsection{Saturation energy and saturation density}

The nuclear binding energy is well described by the semi-empirical mass formula, that is,

$$
B(N, Z)=a_{V} A-a_{S} A^{2 / 3}-a_{A} \frac{(N-Z)^{2}}{A}-a_{C} \frac{Z^{2}}{A^{1 / 3}}+\Delta B,
$$

where the coeffecients $a_{V}, a_{S}, a_{A}$, and $a_{C}$ are the volume, surface, asymmetry and Coulomb coefficient, respectively, while the last term $\Delta B$ is the pairing term. It reads

$$
\Delta B=\left\{\begin{aligned}
\Delta \text { for } Z & =\text { even and } N=\text { even } \\
0 \text { for } A & =\text { odd, } \\
-\Delta \text { for } Z & =\text { odd and } N=\text { odd }
\end{aligned}\right.
$$

and it gives to the even-even nuclei an extra binding contribution $\Delta B$ with respect to the odd ones: this is the reason why, in Sec. 2.1, we have advocated the necessity of using two-particle separation energy to isolate the shell effects starting from the smooth behaviour that is inherent in Eq. (5). Pairing will be discussed in Sec. 5 below.

When we mention saturation, in the nuclear context, we refer to the fact that, excluding very light nuclei, $B / A$ is known to reach an approximately constant value around $8 \mathrm{MeV}$, as it can be easily seen in the left panel of Fig. 2 . In this regime the volume energy per particle $a_{V}$, which is around $16 \mathrm{MeV}$, is reduced to about one half by the other contributions.

Not only the energy saturates, but the bulk density as well. The experimental evidence is that nuclei display, in the interior, an approximately constant density $\rho_{0} \approx 0.16 \mathrm{fm}^{-3}$. This is called the saturation density. In the right panel of Fig. 2 we show the matter densities for a couple of nuclei, and the saturation density is marked by the horizontal line. Nuclear surface, albeit very important for a host of nuclear properties, has a thickness of only $\approx 0.6 \mathrm{fm}$. The interparticle distance $2 r_{0}$ (where $r_{0}$ is defined by $\frac{1}{\rho_{0}}=\frac{4}{3} \pi r_{0}^{3}$ ) is larger than the range of the nuclear force which is between 1 and $2 \mathrm{fm}$. In this respect, the nucleus can be seen as dilute and this fact, together with the role played by the Pauli exclusion principle, explains why nucleons display independent particle motion as discussed in the previous subsection, although the is system governed by the strong force.

Thus, one of the prominent features displayed by medium-heavy nuclei is that their inner part resembles bulk symmetric nuclear matter (medium-heavy nuclei have neutron excess but this is localized at the surface). If we consider this idealized system, i.e. symmetric nuclear matter or SNM, all terms of the mass formula vanish except the volume term $a_{V}$, that gives a value for the energy per particle $E / A$ (equal to $-B / A$ ) of about $-16 \mathrm{MeV}$. In other words, the so-called saturation point $\left(\rho_{0}, E / A\right)=\left(0.16 \mathrm{fm}^{-3},-16 \mathrm{MeV}\right)$ is an equilibrium point for SNM, where $E / A$ as a function of the density has a minumum, and any sensible theory must start from the reproduction of this saturation point.

It turns out that this is not a trivial task. The evidence of independent particle motion would lead us to think that Hartree-Fock (HF) with a properly adjusted effective interaction $V_{\text {eff }}$ might do the job. In reality, such naïve mean field theory fails as discussed in the literature. A simple argument is presented at the start of Ref. [21], while a thorough analysis can be found in Ref. [22]. The failure of a naïve mean field model and the need of DFT for nuclei is 
also the subject of part of the lecture notes [15]. In fact, HF calculations have been carried out from the start using only density-dependent interactions; the density dependence implies that these are not fundamental forces and can naturally be seen merely as generators of an energy density functional, as we explain in what follows.

\section{Density Functional Theory}

Nuclei are quantum many-fermion systems, and let us introduce an Hamiltonian of general form,

$$
H=\sum_{i=1}^{N}-\frac{\hbar^{2}}{2 m} \nabla_{i}^{2}+\frac{1}{2} \sum_{i \neq j=1}^{N} V(i, j),
$$

where $m$ is the nucleon mass ${ }^{2}$, the first term represents the kinetic energy and the second term is a two-body interaction in which $i$ is a shorthand notation for the space coordinate, $\boldsymbol{r}_{i}$, the spin coordinate and any further degree of freedom (e.g. isospin). Three- or four-body interactions are dropped here for the sake of simplicity but their presence does not break the arguments we shall develop. The quantum many-particle systems is hard to treat as we stated in the Introduction but, among the many possible srategies, DFT stands out for its conceptual elegance.

DFT is grounded in the theorem that bears the name of P. Hohenberg and W. Kohn (already referred to as HK), and that has been introduced in their seminal paper [23]. The theorem is valid for any system governed by the Hamiltonian (7), to which an external arbitrary potential $v_{\text {ext }}$ can be added: it basically tells that we can express all ground-state observables in terms of the one-body density only. The one-body density reads

$$
\rho(\boldsymbol{r})=\frac{1}{N} \int d^{3} r_{2} \ldots d^{3} r_{N} \Psi^{*}\left(\boldsymbol{r}_{1} \ldots \boldsymbol{r}_{N}\right) \Psi\left(\boldsymbol{r}_{1} \ldots \boldsymbol{r}_{N}\right)
$$

where $\Psi$ is the $N$-body wave function. This latter depends on $3^{N}$ coordinates while the density depends on 3 coordinates only, hence the advantage of expressing everything in terms of density is very clear!

According to the HK theorem, the total energy $E$, or the energy density $\mathcal{E}$, can be written as a functional of the density, that is,

$$
E_{v_{\mathrm{ext}}}[\rho]=\int d^{3} r \mathcal{E}[\rho(\boldsymbol{r})]=\left\langle\Psi\left|T+V+v_{\mathrm{ext}}\right| \Psi\right\rangle=F[\rho]+\int d^{3} r v_{\mathrm{ext}}(\boldsymbol{r}) \rho(\boldsymbol{r})
$$

This explains the name of Energy Density Functional, or EDF. The latter equality singles out the contribution of the external potential, so that a universal functional $F$ for fermions is implicitly defined. HK have been also able to show that the functional (9) has a minimum at the exact ground-state density, where it assumes the exact energy as a value. The original proof of [23] is limited to non-degenerate ground-states. Extensions of the theorem to the case of degenerate ground states, spin-polarized systems, finite temperature etc. can be found (see [24] and references therein).

Unfortunately, the proof of the HK theorem is not a constructive proof. Showing that the functional exists does not give any clue on how to build it. As we show below, if we generate a functional $E$ by letting an Hamiltonian with the kinetic term $T$ and a two-body force $V_{\text {eff }}$ acting on an indipendent-particle state $|\Phi\rangle$, we obtain a term in $\mathcal{E}$ that goes like $\sim \rho^{2}$. Similarly, a three-body term in the Hamiltonian provides a term $\sim \rho^{3}$ in $\mathcal{E}$. However, as we mentioned above, such simple functionals that emerge from a HF picture are not successful: in other words, there is no need at all to imagine that realistic functionals are generated by acting with simple operators on independent-particle states.

A power series in $\rho$ can be advocated as a mathematically sound and effective approximation to build a functional [25]; however, the appearance of fractional powers of the density is conceivable and these powers can actually mimic many-body correlations. Moreover, assuming that the energy density $\mathcal{E}$ at a given point depends only on the density at the same point is a strong approximation (named Local Density Approximation, or LDA). Non-local effects are to be expected. The fact that $\mathcal{E}$ in $\boldsymbol{r}$ may depend on values of the density at different points $\boldsymbol{r}^{\prime}$, yet around $\boldsymbol{r}$, can be introduced by expanding $\rho\left(\boldsymbol{r}^{\prime}\right)$ in terms of $\rho(\boldsymbol{r})$ : that is, by introducing a dependence on $\mathcal{E}$ on the gradient $\boldsymbol{\nabla} \rho(\boldsymbol{r})$ (gradient approximation) or higher densities. This idea is present in the original paper by Hohenberg and Kohn [23].

While a discussion of the increasingly complex approximations including gradients is doable with reference to the case of electronic systems (see e.g. Ref. [26]), we shall rather examine the nuclear case in detail in the next Section. We first introduce the Kohn-Sham scheme, to show that this is actually the path along which nuclear physics has been moving so far, under the somewhat misleading name of self-consistent mean-field.

${ }^{2}$ The difference between neutron and proton masses is very small and can be neglected here. 


\subsection{The Kohn-Sham scheme}

In the Kohn-Sham (KS) scheme [27], it is assumed that the density $\rho$ can be represented in terms of single-particle wave functions (orbitals) $\phi_{j}(\boldsymbol{r})$, that is,

$$
\rho(\boldsymbol{r})=\sum_{j}\left|\phi_{j}(\boldsymbol{r})\right|^{2}
$$

The index $j$ labels the orbitals. The KS orbitals are, in principle, unrelated to the actual single-particle wave functions (and yet they might be reasonably close to them in specific instances). Within the KS scheme, the total kinetic energy is written as its independent-particle counterpart, that is,

$$
T=\sum_{j}\left\langle j\left|\frac{-\hbar^{2}}{2 m} \nabla^{2}\right| j\right\rangle=\sum_{j} \int d^{3} r \phi_{j}^{*}(\boldsymbol{r})\left(-\frac{\hbar^{2}}{2 m} \nabla^{2}\right) \phi_{j}(\boldsymbol{r})=\int d^{3} r \frac{\hbar^{2}}{2 m} \tau(\boldsymbol{r}),
$$

where

$$
\tau(\boldsymbol{r})=\sum_{j}\left|\nabla \phi_{j}(\boldsymbol{r})\right|^{2}
$$

can be called kinetic energy density and the last equality in (11) comes from an integration by parts. Once again, this is an assumption and does not necessarily mean that the real kinetic energy is accurately represented in this way. The difference between the true (correlated) kinetic energy and the values predicted by the ansatz (11) is supposed to be absorbed in the rest of the functional. The energy functional (9) becomes

$$
E[\rho]=T+V[\rho]+\int d^{3} r v_{\text {ext }}(\boldsymbol{r}) \rho(\boldsymbol{r}),
$$

where $T$ is given in (11).

The key point here is that, having introduced the representation (10), the energy must be minimised, to find the ground-state of the system, with a constraint corresponding to the orthonormality of the orbitals. That is, the equation that defines the costrained minimum of the energy reads

$$
\frac{\delta}{\delta \rho(\boldsymbol{r})}\left(E-\varepsilon \int d^{3} r^{\prime} \phi_{j}^{*}\left(\boldsymbol{r}^{\prime}\right) \phi_{j}\left(\boldsymbol{r}^{\prime}\right)\right)=0
$$

where the symbol $\delta$ is used for the functional derivative, and a Lagrange multiplier $\varepsilon$ is introduced. Elementary introductions to functional derivatives can be found in several textbooks (cf. also Sec. 3.2 of [28]). In the present context we may just need to recall simple rules like

$$
\begin{aligned}
& F[\rho]=\int d^{3} r f(\boldsymbol{r}) \rho(\boldsymbol{r}) \Rightarrow \frac{\delta F}{\delta \rho(\boldsymbol{r})}=f(\boldsymbol{r}), \\
& F[\rho]=\int d^{3} r g(\rho) \Rightarrow \frac{\delta F}{\delta \rho(\boldsymbol{r})}=\frac{\partial g}{\partial \rho}(\boldsymbol{r}) .
\end{aligned}
$$

The reader may note that these rules are understandable as a generalization of the rules for the partial derivatives if the correspondence between the continuous label $\boldsymbol{r}$ and a discrete index is made. In particular, the second equation comes from the chain rule for derivatives. Because, again, of Eq. (10), the functional derivative with respect to $\rho(\boldsymbol{r})$ is equivalent to the derivative with respect to the orbitals $\phi_{j}^{*}(\boldsymbol{r})$. By applying these rules, from Eqs. (14), (13) and (11), one obtains the so-called Kohn-Sham equations:

$$
\left(-\frac{\hbar^{2}}{2 m} \nabla^{2}+v_{\mathrm{KS}}(\boldsymbol{r})+v_{\mathrm{ext}}(\boldsymbol{r})\right) \phi_{j}(\boldsymbol{r}) \equiv h \phi_{j}(\boldsymbol{r})=\varepsilon \phi_{j}(\boldsymbol{r}),
$$

where $v_{\mathrm{KS}}(\boldsymbol{r})=\frac{\delta V}{\delta \rho(\boldsymbol{r})}$ is the Kohn-Sham effective potential, which is not a mean-field potential as it takes correlations implicitly into account. The system (16) is a set of Schrödinger-like equations. Then, the quantities $\varepsilon$ are seen to be the energies associated with the orbitals. The reader should note that the total energy is not the sum of these auxiliary energies (this is left as an exercise).

Fig. 3 shows schematically the Kohn-Sham idea. Instead of solving the interacting system, one deals with a fictitious system of non-interacting, independent particles with the same density. The Kohn-Sham potential $v_{\mathrm{KS}}(\boldsymbol{r})$ is the effective potential in which these particles move. 


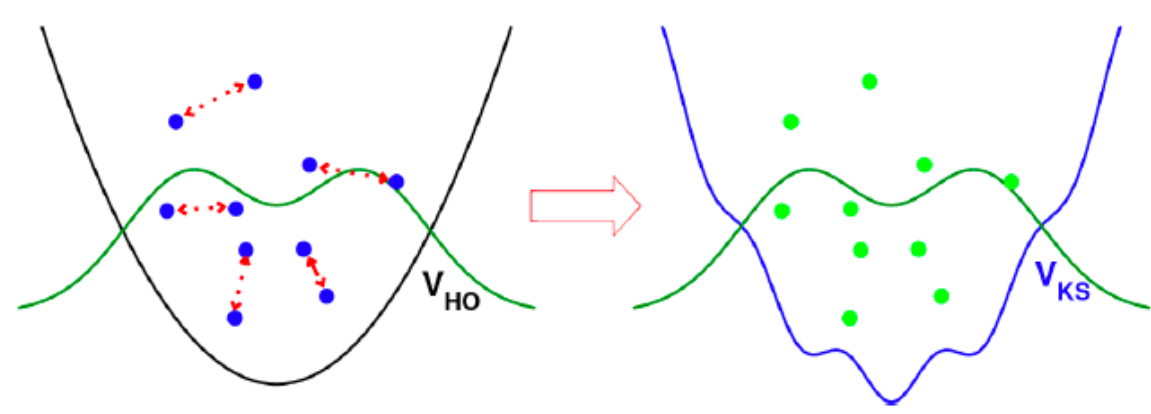

Fig. 3. Schematic picture of the Kohn-Sham approach: in the left picture, interacting particles (blue) are shown in an external potential with the harmonic oscillator shape (black); in the right picture, this system is mapped onto an auxiliary system of non-interacting particles (green) in a Kohn-Sham effective potential (blue). The particle density (green line) is the same. Figure taken from Ref. [29]

\section{Nuclear Energy Density Functionals (EDFs)}

\subsection{An example: a simplified Skyrme functional}

We start from a few words of history and an example which is designed so that one can grasp the main ideas, postponing more general arguments to the next subsection. Nuclear DFT was born as self-consistent mean-field. The idea was, and still sometimes is, to start from an effective interaction $V_{\text {eff }}$ that possesses enough simplicity and flexibility and build the total energy as the expectation value of $H_{\text {eff }}=T+V_{\text {eff }}$ on a mean-field state, namely and independent particle wave function. A reasonably sound $V_{\text {eff }}$ should be short-range, strongly spin- and isospin-dependent, and include a spin-orbit component. It should account for nuclear saturation as a first requirement. In the 1950s, T.H.R. Skyrme was led to propose, for simplicity, a zero-range effective interaction of the type $[30,31]$

$$
\begin{aligned}
V_{\text {Skyrme }}\left(\boldsymbol{r}_{1}, \boldsymbol{r}_{2}\right) & =t_{0}\left(1+x_{0} P_{\sigma}\right) \delta\left(\boldsymbol{r}_{1}-\boldsymbol{r}_{2}\right)+\frac{1}{2} t_{1}\left(1+x_{1} P_{\sigma}\right)\left(\boldsymbol{k}^{\dagger 2} \delta\left(\boldsymbol{r}_{1}-\boldsymbol{r}_{2}\right)+\delta\left(\boldsymbol{r}_{1}-\boldsymbol{r}_{2}\right) \boldsymbol{k}^{2}\right) \\
& +t_{2}\left(1+x_{2} P_{\sigma}\right) \boldsymbol{k}^{\dagger} \cdot \delta\left(\boldsymbol{r}_{1}-\boldsymbol{r}_{2}\right) \boldsymbol{k}+\frac{1}{6} t_{3}\left(1+x_{3} P_{\sigma}\right) \delta\left(\boldsymbol{r}_{1}-\boldsymbol{r}_{2}\right) \rho^{\alpha}\left(\frac{\boldsymbol{r}_{1}+\boldsymbol{r}_{2}}{2}\right) \\
& +i W_{0}\left(\sigma_{1}+\sigma_{2}\right) \cdot \boldsymbol{k}^{\dagger} \times \delta\left(\boldsymbol{r}_{1}-\boldsymbol{r}_{2}\right) \boldsymbol{k}
\end{aligned}
$$

(cf. also Refs. $[32,33]$ ). Here, $P_{\sigma}$ is the spin-exchange operator, $P_{\sigma}=\frac{1+\boldsymbol{\sigma}_{1} \cdot \boldsymbol{\sigma}_{2}}{2}$, and $P_{\tau}$ is the isospin-exchange operator that has an analogous form. These introduce the spin and isospin dependence that we have just mentioned. Zero-range is a simplification, obviously, but some amount of non-locality is brought by the momentum dependence: in fact, $\boldsymbol{k}=-\frac{i}{2}\left(\nabla_{1}-\nabla_{2}\right)$ is the relative momentum operator acting at right and $\boldsymbol{k}^{\dagger}$ is the adjoint operator acting at left. Although we do not dwell on the reason here, the last term is indeed a spin-orbit term. In the force (17), there are 10 parameters to be fitted, namely $t_{i}, x_{i}, \alpha$ and $W_{0}$.

One can now build an $\operatorname{EDF} E[\rho]$ by means of

$$
E[\rho]=\left\langle\Phi\left|T+V_{\mathrm{eff}}\right| \Phi\right\rangle
$$

where $|\Phi\rangle$ is the most general Slater determinant, made up with single-particle wave functions $\phi_{j}$, and consistent with the symmetries of the system. Eventually, a force like (17) is hard to be interpreted as a true potential because of its density-dependence. Originally, it has been named as pseudo-potential and this wording has been proposed again in recent years. The rationale is that it is not the potential that matters, but the associated EDF (18). Now, practitioners build new EDFs directly [34,35], and if one wishes to start from an effective $V_{\text {eff }}$ this is just an alternative, more specific and less general, route. Potentials are just, in this context, ways to generate an EDF. Hereafter, we use the name Skyrme EDF both for functionals generated via a potential and for more general local EDFs.

As a pedagogical exercise, we can show the reader that even a simplified Skyrme EDF, obtained via a simplified $\left(t_{0}, t_{3}\right)$ Skyrme force, without momentum and spin dependence, can produce nuclear saturation in symmetric nuclear matter. We start from

$$
V\left(\boldsymbol{r}_{1}, \boldsymbol{r}_{2}\right)=t_{0} \delta\left(\boldsymbol{r}_{1}-\boldsymbol{r}_{2}\right)+\frac{1}{6} t_{3} \delta\left(\boldsymbol{r}_{1}-\boldsymbol{r}_{2}\right) \rho^{\alpha}\left(\frac{\boldsymbol{r}_{1}+\boldsymbol{r}_{2}}{2}\right)
$$




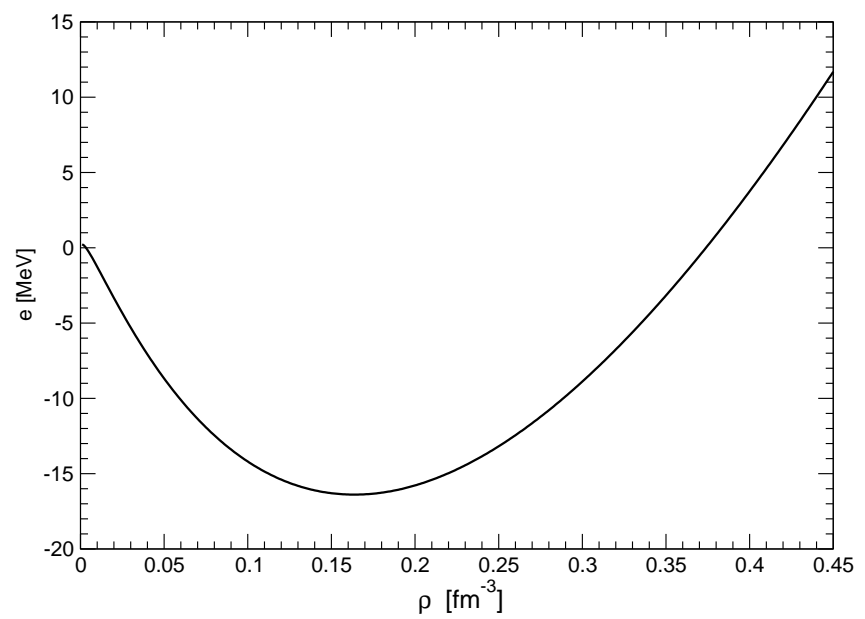

Fig. 4. Energy per particle $e \equiv E / A$ in symmetric nuclear matter, calculated with a simplified Skyrme functional of the type (19) and parameters $t_{0}=-2552.84 \mathrm{MeV} \cdot \mathrm{fm}^{3}, t_{3}=16694.7 \mathrm{MeV} \cdot \mathrm{fm}^{3(\alpha+1)}, \alpha=0.20309$.

and we show in the Appendix that this generates, for even-even systems, the functional

$$
\begin{aligned}
& E\left[\rho_{n}, \rho_{p}\right]=\int d^{3} r \mathcal{E}\left[\rho_{n}, \rho_{p}\right], \\
& \mathcal{E}\left[\rho_{n}, \rho_{p}\right]=\frac{\hbar^{2}}{2 m} \tau+\frac{1}{2} t_{0}\left[\rho^{2}-\frac{1}{2}\left(\rho_{n}^{2}+\rho_{p}^{2}\right)\right]+\frac{1}{12} t_{3}\left[\rho^{\alpha+2}-\frac{1}{2} \rho^{\alpha}\left(\rho_{n}^{2}+\rho_{p}^{2}\right)\right] .
\end{aligned}
$$

We can apply this simplified functional in SNM, where densities are uniform (that is, numbers and not functions) and $\rho_{n}=\rho_{p}=\rho / 2$. In uniform matter the orbitals $\phi_{j}$ are plane waves due to translational invariance, namely

$$
\phi_{\boldsymbol{k}}(\boldsymbol{r})=\frac{1}{(2 \pi)^{3 / 2}} \exp (i \boldsymbol{k} \cdot \boldsymbol{r})
$$

The sum over $j$ in Eqs. (10) and (12) is replaced by an integral and leads to the well-known results known from the case of the Fermi gas,

$$
\begin{aligned}
\rho_{q} & =2 \frac{1}{(2 \pi)^{3}} \int_{0}^{k_{F}} d^{3} k 1=\frac{k_{F}^{3}}{3 \pi^{2}}, \\
\tau_{q} & =2 \frac{1}{(2 \pi)^{3}} \int_{0}^{k_{F}} d^{3} k k^{2}=\frac{k_{F}^{5}}{5 \pi^{3}},
\end{aligned}
$$

where the factor 2 at r.h.s. refers to the spin degeneracy. Thus, in uniform matter $\tau_{q}=\frac{3}{5}\left(3 \pi^{2}\right)^{2 / 3} \rho_{q}^{5 / 3} \equiv C \rho_{q}^{5 / 3}$. Then,

$$
\begin{aligned}
& E_{\mathrm{SNM}}[\rho]=\int d^{3} r \frac{\hbar^{2}}{2 m} \frac{C}{2^{2 / 3}} \rho^{5 / 3}+\frac{3}{8} t_{0} \rho^{2}+\frac{1}{16} t_{3} \rho^{\alpha+2}, \\
& \frac{E}{A}=\frac{\mathcal{E}}{\rho}=\frac{\hbar^{2}}{2 m} \frac{C}{2^{2 / 3}} \rho^{2 / 3}+\frac{3}{8} t_{0} \rho+\frac{1}{16} t_{3} \rho^{\alpha+1} .
\end{aligned}
$$

The two parameters $t_{0}, t_{3}$, once $\alpha$ is fixed, can be fit in such a way that the function $E / A$ has a minimum at the saturation point $\left(\rho_{0}, E_{0} / A\right)=\left(0.16 \mathrm{fm}^{-3},-16 \mathrm{MeV}\right)$. An example of such a fit is provided in Fig. 4. Alternatively, one could fit the three parameters on the values of the saturation point and the curvature $K_{\infty}$ at that point [cf. Eq. (50) below].

\subsection{Modern realistic functionals}

Skyrme functionals have become much more involved than the simple one we have just introduced for pedagogical reasons. Because of the zero-range character of the force, Skyrme interactions will always produce a local functional, 
that is, an energy density that depends on densities at the very same point. In this respect, they constitute the prototype of a general local functional in the nonrelativistic case.

The most general local functional will depend on all possible local densities. These can be built in a systematic way, as described in Refs. [33,36] (see also Sec. 7.3 .4 of [11]). For spin-1/2 fermions the non-local density is a $2 \times 2$ matrix that can be expanded as

$$
\rho_{\alpha \beta}\left(\boldsymbol{r}, \boldsymbol{r}^{\prime}\right)=\rho\left(\boldsymbol{r}, \boldsymbol{r}^{\prime}\right) \mathbb{1}+\boldsymbol{s}\left(\boldsymbol{r}, \boldsymbol{r}^{\prime}\right) \cdot \boldsymbol{\sigma},
$$

where the Pauli matrices $\boldsymbol{\sigma}$ form a complete basis for $2 \times 2$ matrices together with the identity matrix $\mathbb{1}$. Derivative operators can be applied in a systematic way, by stopping at a given order. Up to second order, the procedure is described in Ref. [36]. It is mandatory to start from the non-local quantities defined by Eq. (24) because derivative operators can act either on $\boldsymbol{r}$ or $\boldsymbol{r}^{\prime}$; after applying the operators, one moves to local quantities, namely to local density $\left.\rho\left(\boldsymbol{r}, \boldsymbol{r}^{\prime}\right)\right|_{\boldsymbol{r}=\boldsymbol{r}^{\prime}}$ and local spin density, $\left.\boldsymbol{s}\left(\boldsymbol{r}, \boldsymbol{r}^{\prime}\right)\right|_{\boldsymbol{r}=\boldsymbol{r}^{\prime}}$. Since the functional must be invariant under space translations, only derivatives with respect to the relative coordinate, $\boldsymbol{\nabla}-\boldsymbol{\nabla}^{\prime}$, must be considered. Due to the identity

$$
\left(\boldsymbol{\nabla}-\boldsymbol{\nabla}^{\prime}\right)^{2}=\left(\boldsymbol{\nabla}+\boldsymbol{\nabla}^{\prime}\right)^{2}-4 \boldsymbol{\nabla} \cdot \boldsymbol{\nabla}^{\prime}
$$

the action of the second derivative boils down to that of the operator $\nabla \cdot \nabla^{\prime}$. Starting from $\rho$, therefore, one obtains the local current

$$
\boldsymbol{j}(\boldsymbol{r})=\frac{1}{2 i}\left[\left(\boldsymbol{\nabla}-\boldsymbol{\nabla}^{\prime}\right) \rho\left(\boldsymbol{r}, \boldsymbol{r}^{\prime}\right)\right]_{\boldsymbol{r}=\boldsymbol{r}^{\prime}}
$$

and the kinetic energy density

$$
\tau(\boldsymbol{r})=\left[\boldsymbol{\nabla} \cdot \nabla^{\prime} \rho\left(\boldsymbol{r}, \boldsymbol{r}^{\prime}\right)\right]_{\boldsymbol{r}=\boldsymbol{r}^{\prime}}
$$

that has been alread introduced in Eq. (12). It is easy to derive the corresponding derivatives of the spin density. It has to be noted that one has to deal with both neutron and proton densities, so that the number of densities is doubled at each step (cf. [33,36], or Sec. I.5 of Ref. [9]).

Once all possible local density are defined, the most general EDF will be a combination of scalar terms that depend on these densities, and are invariant under parity and time-reversal. Even if one limits oneself to terms that are quadratic in the densities, the number of these terms can grow up dramatically if one allows higher-order derivatives (cf. Ref. [37], and in particular Table XXVII therein). In front of each term one has a parameter that either is a number, or that could be itself a function of the density. If the structure of the EDF is too involved, fitting all parameters becomes practically impossible. Gauge invariance may be imposed to reduce the number of terms, but the meaning of this invariance has still to be clarified [38]. One should also ask the question whether all parameters are actually uncorrelated; recently, correlation analysis has become a tool that EDF practitioners use more and more [39].

There exist functionals that are not quadratic in the local densities [40,41]. Also, non local-functionals can be obviously conceived. In practice, the most widely used non-local EDFs are derived from the Gogny effective interaction or pseudo-potential [through Eq. (18)]. The Gogny interaction reads $[42,43]$

$$
\begin{aligned}
V_{\text {Gogny }}\left(\boldsymbol{r}_{1}, \boldsymbol{r}_{2}\right) & =\sum_{j=1}^{2} e^{\frac{\left|\boldsymbol{r}_{1}-\boldsymbol{r}_{2}\right|^{2}}{\mu_{j}^{2}}}\left(W_{j}+B_{j} P_{\sigma}-H_{j} P_{\tau}-M_{j} P_{\sigma} P_{\tau}\right) \\
& +t_{3}\left(1+x_{0} P_{\sigma}\right) \delta\left(\boldsymbol{r}_{1}-\boldsymbol{r}_{2}\right) \rho^{\alpha}\left(\frac{\boldsymbol{r}_{1}+\boldsymbol{r}_{2}}{2}\right)+i W_{l s}\left(\boldsymbol{\sigma}_{1}+\boldsymbol{\sigma}_{2}\right) \cdot \boldsymbol{k}^{\dagger} \times \delta\left(\boldsymbol{r}_{1}-\boldsymbol{r}_{2}\right) \boldsymbol{k},
\end{aligned}
$$

where the notation is the same as above for the Skyrme force (17). This interaction is the sum of two Gaussians with exchange operators, a density-dependent term and a spin-orbit term, and has 14 free parameters to be adjusted. Effective forces of Yukawa-type have been also used to derive EDFs [44].

We do not dwell too much here on relativistic, or covariant, functionals; as stated in the Introduction, excellent review paper exist. It is worth mentioning that they started as EDFs generated through effective Lagrangians including nucleons treated as Dirac particles, effective mesons (like the scalar-isoscalar and vector-isoscalar $\sigma$ and $\boldsymbol{\omega}$ mesons, plus the vector-isovector $\boldsymbol{\rho}$ meson), and the mutual meson-nucleon couplings. Recently, they are also built directly without reference to such underlying Lagrangians. One starts by considering the Dirac single-particle spinors $\psi$ instead of the orbitals that have been introduced in Eq. (10). There exist, then, 16 covariant local quantities of the type

$$
\bar{\psi}(\boldsymbol{r}) \Gamma_{i} \psi(\boldsymbol{r}), \quad i=1 \ldots 16
$$

that can be considered as densities to start from. $\Gamma=1$ and $\Gamma=\gamma^{\mu}$ provide the familiar scalar and vector densities but one can complete the classification $[9,12,13]$. The most general covariant EDF will be a combination of all possible terms that are built with the covariant local densities, and respect symmetries.

Despite technical differences, there is not a deep difference between local and non-local functionals, probably due to the short-range nature of the $\mathrm{NN}$ force. At the start of the millennium, some debate has taken place concerning the 


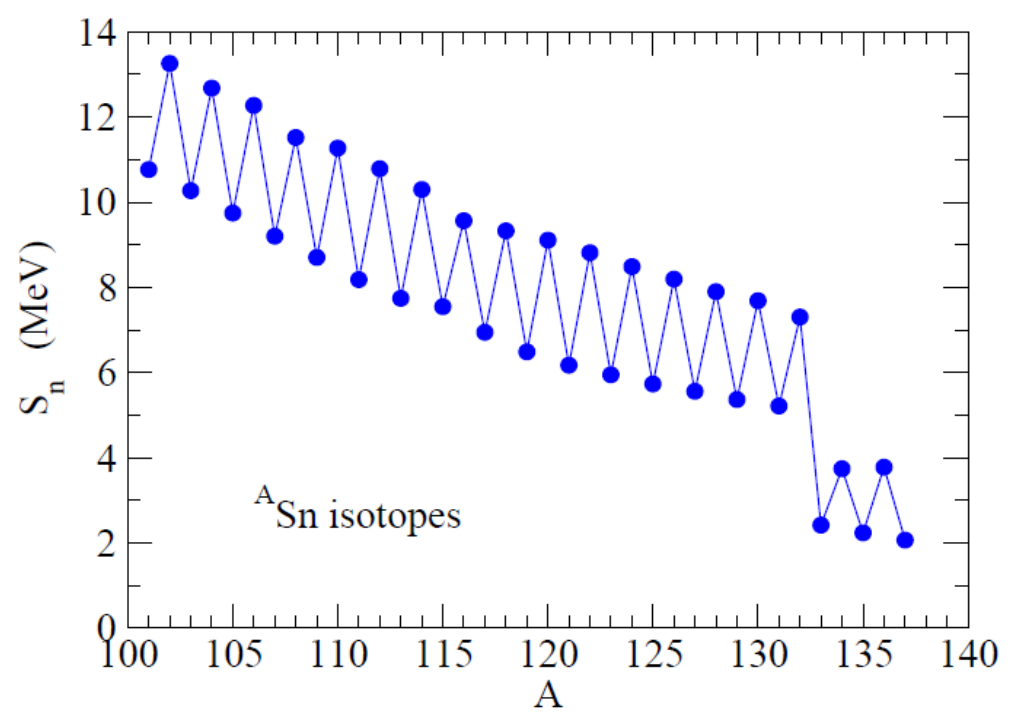

Fig. 5. Separation energy of the Sn isotopes. We remind that $S_{n}=B E(N+1)-B E(N)$.

difference between nonrelativistic and covariant EDFs. Although nucleons can have expectation values of the singleparticle momentum that correspond to non-negligible values of $v / c(\approx 0.2-0.3)$, there is no clear difference between the performance of the two classes of EDFs. If calibrated with similar fitting protocols, they provide comparable results. This points to the fact that parameters are likely to absorb the kinematical differences. One advantage of covariant functionals is that the spin-orbit terms come out naturally from the Dirac scheme.

In the next Sections, we shall discuss some examples of applications. The interested readers can see many more examples in the recent literature (see, e.g., Ref. [45] and references therein).

\section{Nuclear superfluidity and EDFs}

There are several evidences that part of the nucleons in the nuclear medium may form Cooper pairs like in a superfluid [46]; while we describe below those which are relevant in the framework of this lecture, further ones like the moment of inertia of deformed nuclei [47] should not be overlooked.

One of the main evidences is the trend of the binding energies, in particular the so-called odd-even staggering. Even in the simple semi-empirical formula (5), as we have already stressed, the last term at the r.h.s. is the pairing term that gives to even-even nuclei an extra binding energy $\Delta$ with respect to odd nuclei [cf. Eq. (6) and the discussion around it]. In other terms, nucleons tend to form pairs and gain energy from such pairing correlations. The value of $\Delta$ for stable nuclei is $\approx 12 / \sqrt{A}[\mathrm{MeV}]$, but there is no reason, in the spirit of the title of this volume, to believe that this is the case for exotic systems. We shall come back to this point in the last part of this Section.

Specific filters can be, and have been, introduced to pin down quantitatively the pairing correlations ${ }^{3}$. In particular, the widely used three-point formula for the neutron pairing gap reads

$$
\Delta_{n}^{(3)}(N, Z)=(-)^{A+1} \frac{B(N+1, Z)-2 B(N, Z)+B(N-1, Z)}{2}=(-)^{A+1} \frac{S_{n}(N+1, Z)-S_{n}(N, Z)}{2} .
$$

A similar formula holds, evidently, for the case of protons. This quantity is expected to be proportional to the last term $\Delta$ in Eq. (5). The last equality in (28) shows that a non-zero value for the pairing gap is reflected in the trend of the separation energies. This can be seen in Fig. 5 for the case of Sn isotopes.

Not only ground-state energies but also excitation spectra bring evidence for pairing correlations. Even-even nuclei display an energy gap between the ground state and the first excited state (hence the above name of gap for the quantity $\Delta$ ), while this is not the case for the odd nuclei. This fact suggests that the nucleon pairs must be broken, in the former case of even-even nuclei, to excite the system; this is not true in the odd system where one nucleon is not paired.

This gap in the excitation system reminds the case of superconducting metals and led, historically, to the first applications of the Bardeen-Cooper-Schrieffer (BCS) theory of superconductivity to atomic nuclei [50]. Within BCS,

\footnotetext{
${ }^{3}$ Subtle interferences between pairing effects and different effects are discussed in Refs. [48,49].
} 
one introduces a wave function which is more general than the Slater determinant $|\Phi\rangle$ that has been introduced in Sec. 4.1. This wave function, made up with a set on orbitals $j$, correspond to occupying the lowest among those orbitals. If we assume time-reversal invariance so that the orbital $j$ and its time-reversed $\tilde{j}$ are either empty or filled together, we can write

$$
|\Phi\rangle=\Pi_{j} a_{j}^{\dagger} a_{\tilde{\jmath}}^{\dagger}|-\rangle
$$

where $a_{j}^{\dagger}$ are single-particle creation operators ( $a$ would be the corresponding annihilation operator) and act on the vacuum $|-\rangle$. The product spans the orbitals $j$ up to the Fermi energy. BCS assumes that the ground-state may be formed by placing pairs of particles on the levels in a more general manner, since the total energy can be minimal if the price paid in setting particles on higher levels can be compensated by pairing correlations as we have hinted above. Thus, the BCS wave function reads

$$
|\mathrm{BCS}\rangle=\Pi_{j}\left(u_{j}+v_{j} a_{j}^{\dagger} a_{\tilde{\jmath}}^{\dagger}\right)|-\rangle
$$

where $u$ and $v$ are additional variational parameters that represent, respectively, the probability amplitude that $j$ is empty or filled. [In the case of Eq. (29), below (above) the Fermi energy $u=0$ and $v=1(u=1$ and $v=0)$ ]. In a spherical nucleus, the orbitals are characterised by the quantum number $m$ that corresponds to the projection of the angular momentum $j$ on the $z$-axis. The BCS wave function (30) includes pairs formed with nucleons on the same orbital, and having respectively $\pm m$. These two states can be easily shown to have total angular momentum $J=0$ and also $L=0, S=0$ (one speaks of $T=1$ or singlet pairing). The Hartree-Fock-Bogoliubov (HFB) $[5,9]$ assumes a more general form than (30), in which all pairs having the quantum numbers we have mentioned show up, regardless of being particles on the same radial orbit or not. HFB becomes the tool of choice in neutron-rich nuclei, where nucleons are close to the continuum and quasi-bound levels play a role for pairing correlations, so that the restrictive BCS assumption becomes questionable.

The minimization of the expectation value

$$
E=\left\langle\mathrm{BCS}\left|T+V_{\mathrm{eff}}\right| \mathrm{BCS}\right\rangle
$$

is, therefore, more general than the minimization of (18). Actually, the BCS or HFB states do not have a fixed particle number as one can see immediately from Eq. (30). In order to constrain the average particle number to a specific value, one has to minimise

$$
E^{\prime}=\left\langle\mathrm{BCS}\left|T+V_{\text {eff }}-\lambda N\right| \mathrm{BCS}\right\rangle,
$$

where $\lambda$ is a Lagrange multiplier that can be seen as $\partial E / \partial N$. The equations to which this procedure leads, can be found in standard textbooks [5]. BCS or HFB equations include the so-called gap equation that provides the state dependent values of $\Delta_{j}$ as a solution. Moreover, in such frameworks, the concept of Kohn-Sham single-particle energy $\varepsilon_{j}$ must be generalized, and one obtains the so-called quasi-particle energies. They are expressed as

$$
E_{j}=\sqrt{\left(\varepsilon_{j}-\lambda\right)^{2}+\Delta_{j}^{2}}
$$

If we assume that the ground state of the odd nucleus is a quasi-particle state on top of the even core, then the comparison between Eqs. (28) and (33) clearly shows that $E_{j} \approx \Delta_{j} \approx \Delta^{(3)}$. From Eq. (33), one also understands clearly why there is a gap in the excitation spectrum of an even-even nucleus.

At the start of BCS theory for nuclei, the equations have been solved with empirical, ad hoc Hamiltonians. Skyrme forces do not give realistic solutions unless they are complemented with specific zero-range terms that are active only in the pairing channel (cf. Fig. 6). On the other hand, the Gogny interaction (26) has a peculiar structure so that it can be used effectively within Eq. (31). The reader should we aware that RMF calculations are, as a rule, performed by including the pairing component of the Gogny force. In order to cast all this into the DFT picture, we come back to the fact that in the BCS (or HFB) case the states $j$ have a non-trivial probability of being empty $\left(u_{j}^{2}\right.$ ) or filled $\left(v_{j}^{2}\right)$. This is reflected in the non-trivial expectation value of $u_{j} v_{j}$, that would be instead zero if evaluated on the Slater determinant $|\Phi\rangle$ of an even-even nucleus. This new degree of freedom calls for an extension of the variables on which an EDF may depend. The usual density can be seen as

$$
\rho(\boldsymbol{r}) \equiv\left\langle a^{\dagger}(\boldsymbol{r}) a(\boldsymbol{r})\right\rangle
$$

where now the expectation value is meant to be on a general state. The non-trivial value of $u v$ can be captured by another type of density that is called abnormal density (or pairing tensor), and reads

$$
\kappa(\boldsymbol{r}) \equiv\langle a(\boldsymbol{r}) a(\boldsymbol{r})\rangle .
$$

In fact, there is a finite probability of destroying or creating two particles and remain in the same state if a wave function like (30) is assumed. The formal proof that the two above densities uniquely determine a BCS or HFB state can be found in Sec. 7.3.2 of Ref. [5]. 


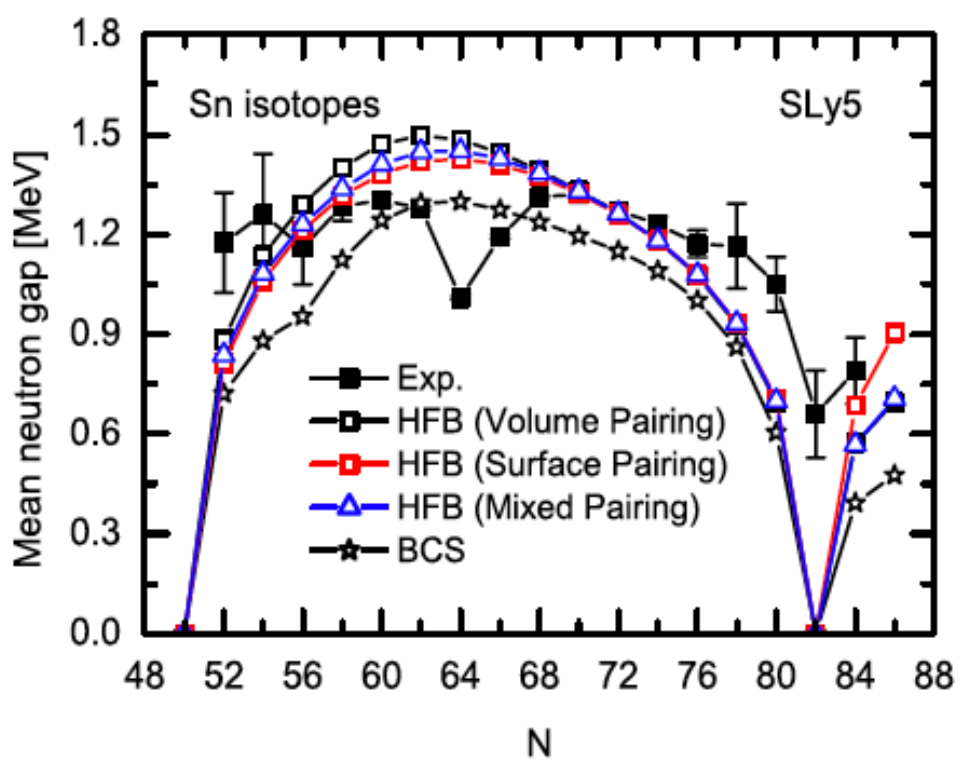

Fig. 6. Pairing gap for the Sn isotopes. The value extracted from the three-point formula (28) are compared with Skyrme calculations with the functional SLy5 [51], in which different kind of pairing forces are introduced, either in the BCS or HFB approach (see the original reference [52] from which the figure is taken for details on these forces).

Thus, we can now assume the existence of an $\operatorname{EDF}$ like $E[\rho, \kappa]$ without reference to an underlying expectation value of whatever effective Hamiltonian. In close analogy with the discussion carried out in 4.1 and in the Appendix, a simple zero-range, two-body pairing force like $V_{0} \delta\left(\boldsymbol{r}-\boldsymbol{r}^{\prime}\right)$ acting between protons and neutrons can be shown to produce a term in the EDF that reads

$$
E_{\text {pair }}=\frac{1}{2} \int d^{3} r V_{0}\left[\kappa_{n}^{2}(\boldsymbol{r})+\kappa_{p}^{2}(\boldsymbol{r})\right]
$$

We do not provide the explicit proof but the result is intuitive. I advise the reader who wishes to understand more deeply to go through Refs. [53,54]. As pairing correlations do depend on density (cf. below), EDFs that assume that the coupling strength $V_{0}$ is a function of $\rho$ are more successful, whereas taking $V_{0}$ as a constant is a crude approximation. This is particularly true for exotic nuclei [55]. Recently, EDFs that depend on the total density and also on $\rho_{n}-\rho_{p}$, have been proposed [56]. In principle, one could go in the direction of extending (36) by including a dependence on derivatives of $\kappa$, or on the non-local pairing tensor $\kappa\left(\boldsymbol{r}, \boldsymbol{r}^{\prime}\right)$. Formally, such extensions have been considered (see, e.g, Ref. [57]); in practice, the values of the pairing gap $\Delta_{n}$ and $\Delta_{p}$ are the only available quantities to fix the dependence of $E$ on $\kappa_{n}$ and $\kappa_{p}$, so that there are no constraints to fix more sophisticated dependences.

The main question at present, as we stressed above, is how pairing evolves far from the stability valley. A new textbook on nuclear physics should be able to replace the simple estimate that we have already alluded to, namely $\Delta \approx 12 / \sqrt{A}[\mathrm{MeV}]$, with something that holds in the broader landscape that includes unstable nuclei. In other words, the dependence of $E$ on $\rho$ and $\kappa$ (plus derivative terms) should be more constrained so that $E$ can have predictive power for the exotic nuclei. Theoretical predictions made with realistic forces for the case of uniform matter suggest that the pairing gap $\Delta$ may increase at low density and take, at around $\rho_{0} / 10$, a value which is considerably larger than that around the normal density. This is clear from Fig. 7, in which the right panel corresponds to the usual neutron-neutron (or proton-proton) case and the gap is of the order of $\approx 1 \mathrm{MeV}$ at the usual density ${ }^{4}$. Accordingly, one can expect enhanced pairing correlations in light, neutron-rich nuclei like ${ }^{6} \mathrm{He}$ and ${ }^{11} \mathrm{Li}$ [61,62]; in fact, such nuclei are characterized by low-density distributions of neutrons around the nuclear surface (the so-called neutron skin and/or neutron halo). A recent analysis predicts also more significant pairing correlations in medium-mass neutron-rich nuclei [63].

We leave aside the subject of pairing in the inner crust of neutron stars and other subjects, like the BCS-BEC crossover, or the possibility of pairing in other channels than $L=0, S=0, J=0$ [64]. Another subject of great interest is whether pairing between protons and neutrons ( $T=0$ or triplet pairing) is strong enough to give rise to

\footnotetext{
4 The discussion of the relationship between calculations carried out with either realistic forces like the Paris potential or Gogny forces, is beyond the scope of this introductory lecture. Ref. [60] is useful in this respect.
} 


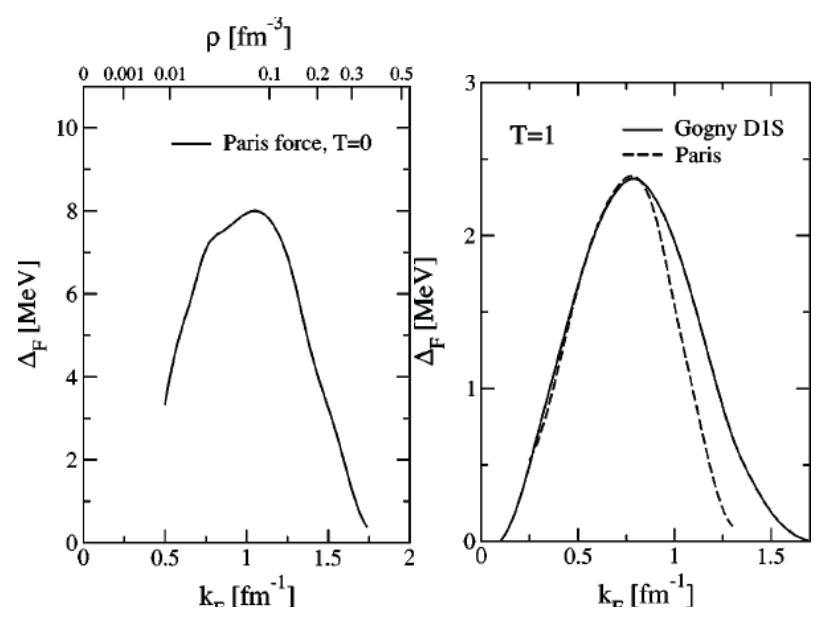

Fig. 7. Fermi momentum dependence of the pairing gaps, for the $T=0$ triplet case and the $T=1$ singlet case. The $T=0$ results are calculated by using the Paris potential [58], while the $T=1$ results are obtained by using either the Paris potential or the Gogny D1S effective force [43]. The corresponding nuclear density is also given in the upper axis of the figure for the $T=0$ case. The figures are taken from Ref. [59].

a condensate. The left panel of Fig. 7 seems to hint this, but the level structure of finite nuclei may disfavour such situation. A recent review in this topic can be found in Ref. [65].

\section{DFT calculations of ground-state nuclear properties}

The main observable that one aims at calculating with an EDF, is the total energy which is actually the binding energy of a nucleus and can be compared with very accurate experimental data. The typical errors are of the order of $\approx 1-2 \mathrm{MeV}$. A recent survey can be found in Table 1 of Ref. [45]. Local nonrelativistic functionals like HFB24 [66] and UNDEF1 [67,68] display a r.m.s. deviation (theory vs. experiment) of $0.549 \mathrm{MeV}$ and $1.88 \mathrm{MeV}$, respectively; the same quantity is $2.01 \mathrm{MeV}$ for the covariant EDF named DD-PC1 and introduced in Ref. [69]. These numbers should be taken with a grain of wisdom. While UNEDF1 and DD-PC1 are true EDFs, HFB24 is a mass model that is mainly based on a local Skyrme ansatz but is supplemented by terms that are outside the DFT philosophy (it has 30 parameters, at variance with the 9-12 parameters of DD-PC1 and UNEDF1). In this respect, one may compare the performance of HFB24 with that of so-called mic-mac (microscopic-macroscopic) models that are characterized by r.m.s. deviations like $0.559 \mathrm{MeV}(\operatorname{FRDM}(2012)$ of Ref. [70]) or $0.298 \mathrm{MeV}$ (WS4 or Ref. [71]).

Having accuracies of the order of $2 \mathrm{MeV}$ may be thought to be small, at least in comparison with total energies that span values like $10^{2}-10^{3} \mathrm{MeV}$. But one should keep in mind that the accuracy in predicting masses is instrumental to obtain the $Q$-values of nuclear reactions of $\beta$-decays: these depend on mass differences that can benefit from error cancellations but also be harmed by their amplification. Thus, the effort of pushing down the error of mass models is still continuing. Needless to say, while a reduction of the error of a mass model does not trigger any improvement on the predition of other observables, in the case of DFT-based models one can look simultaneously at masses and other quantities.

In Fig. 8 we show two examples of detailed comparison between experimental binding energies and the result of DFT calculations. The Skyrme functional SLy4 [51] is shown because it is the best example of Skyrme EDFs of the last millennium: in it, masses and charge radii of basically only magic nuclei are included in the parameter fit. A much more sophisticated fitting protocol has been used to fit UNEDF0 [67], and both spherical and deformed nuclei are included in the fit. While this definitely improves the behaviour of the residuals, these are not really normally distributed. Both panels of Fig. 8 show the arch-like behaviour that are common to many EDFs. The arches span shells: in other words, it seems that, still, reproducing with similar accuracy both closed-shell and open-shell nuclei represents a non-trivial challenge. Some nuclear correlations are still not included in the most up-to-date EDFs.

Along, again, the spirit of this volume, one of the aspects at the forefront of current nuclear physics research is the determination of where the so-called drip lines are located. The one- or two-neutron drip-line is the locus of the nuclear chart where nuclei decay by emitting either one or two neutrons, that is, where either $S_{n}$ or $S_{2 n}$ vanishes. A 

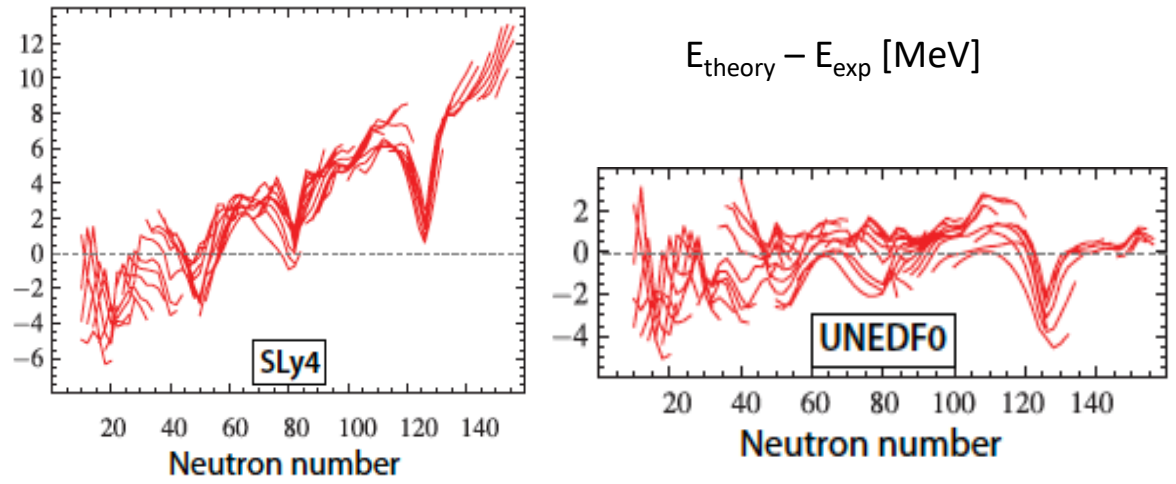

Fig. 8. Two examples of comparison between theoretical and experimental binding energies. The left panel corresponds to the functional SLy4 from Ref. [51] while the right panel to the functional UNEDF0 from Ref. [67]. Figure taken and adapted from Ref. [67].

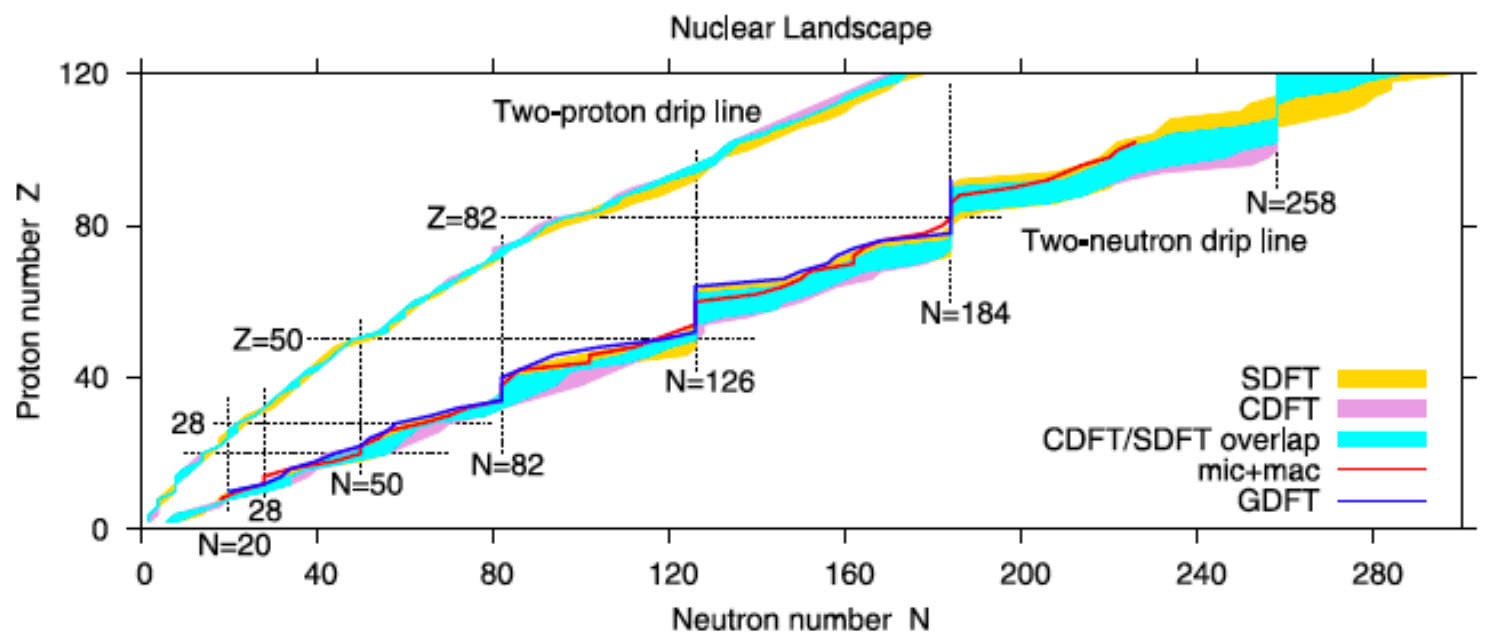

Fig. 9. Two-neutron and two-proton drip lines. See the text for an explanation of the colored bands and lines, as well as for a discussion. Figure taken and adapted from Ref. [72].

similar definition holds for the proton drip line. Beyond the drip lines, nuclei are not bound. Between the region in which nuclei are stable and the drip lines lies the large territory where nuclei are unstable with respect to the weakinteraction as they undergo $\beta$-decay, but can be considered bound systems with respect to the strong interaction. Radioactive beam facilities (RIBFs) are exploring this territory but yet most of the unstable nuclei are unknown. The performance of the current DFT-based models in predicting the drip line location can be judged by inspecting at Fig. 9 as well by studying the orginal papers [72,73]. The two-neutron and two-proton drip lines (with their model uncertainties) have been evaluated using the Skyrme EDF SV-min in Ref. [73] and the corresponding area is labelled as SDFT in Fig. 9 (yellow band). The same lines and uncertainties have been evaluated with covariant state-of-the-art 
$\mathrm{Ca}$

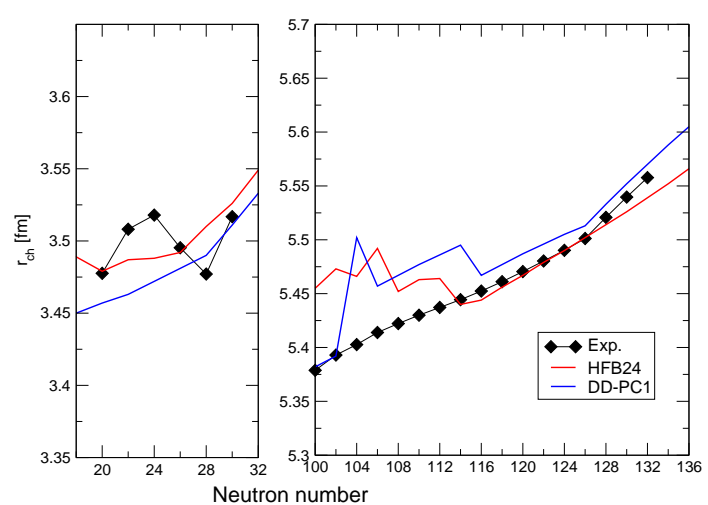

$\mathrm{N}$

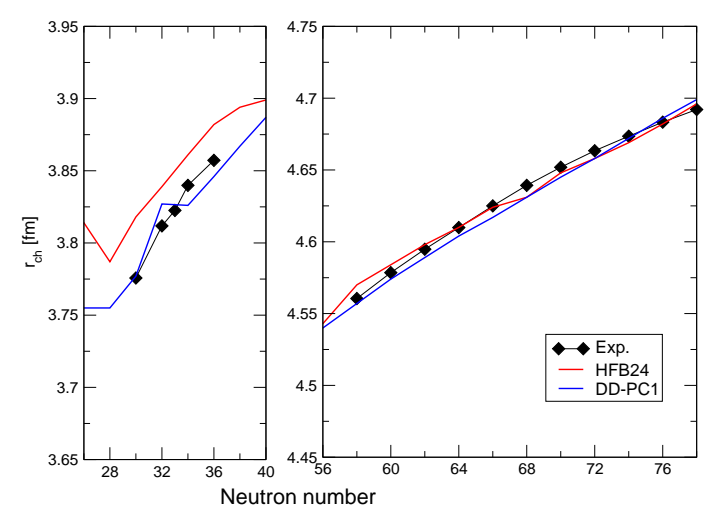

Fig. 10. Comparison of charge radii from experimental measurements and from DFT calculations. The experimental data are from Ref. [76] and are represented by symbols. The lines refer to different calculations, performed either with the nonrelativistic HFB24 mass model [66] or with the covariant functional DD-PC1 introduced in Ref. [69]. See the text for a short discussion.

EDFs in [72] (pink band). EDFs predictions overlap to some extent (light blue band) with each other and/or the predictions of the mic-mac model of Ref. [74] (red line) and the Gogny EDF of Ref. [75] (blue line). However, it is evident from the figure that, especially going towards heavy nuclei, the predictions for the neutron drip line can be markedly model-dependent, and systematic uncertainties may reach $\approx$ ten mass units. Proton drip lines suffer from less uncertainties because they lie closer to the stability valley.

Another quantity, related to the total energy, that testifies to the quality of a given EDF, is the charge radius. In general, one could discuss the neutron and proton r.m.s. radii, which are the square root of

$$
\left\langle r_{q}^{2}\right\rangle \equiv \int d^{3} r \rho_{q}(\boldsymbol{r}) r^{2}
$$

where $q$ labels either neutrons or protons. According to DFT, the exact ground-state should be a solution that is characterised simultaneosly by the correct energy and density; in this respect, EDFs must provide satisfactory results for both energies and neutron/proton radii. Unfortunately, we do not know so well neutron densities and radii so far, because of the lack of a model-independent probe (cf. the discussion in Sec. 5.2.2 of [45], and the references therein). Charge density distributions are available due to electron scattering or, to some extent, laser precision spectroscopy. There exist precise relationships between the charge density r.m.s. radii and the proton density r.m.s. radii, that involve the nucleon electromagnetic form factors; nevertheless, the approximate relationship $\left\langle r_{\text {charge }}^{2}\right\rangle=\left\langle r_{p}^{2}\right\rangle+0.8^{2}\left[\mathrm{fm}^{2}\right]$ has been shown to be accurate enough.

Recalling two of the models that have been introduced above for masses, HFB24 and DD-PC1, they have a r.m.s. deviation on charge radii of $0.025 \mathrm{fm}$ and $0.028 \mathrm{fm}$, respectively [45]. While we said before that the errors on masses follow the arch-like trends as in Fig. 8, the picture is somewhat more blurred for radii. Some examples of comparison of the results of these DFT calculations with experiment are shown in Fig. 10. Starting from heavier nuclei, the two different EDFs have a very small spread, among themselves and with experiment, in the case of the Sn isotopes. In the case of the $\mathrm{Pb}$ isotopes, if we restrict ourselves to neutron numbers $N$ larger than 116, HFB24 is closer to the experimental data in terms of absolute values but DD-PC1 predicts better the kink after $N=128$, which is well recognized since quite some time and is known to be due to the different spin-orbit terms associated with the functionals ${ }^{5}$. In the case of $\mathrm{Ni}$ isotopes, DD-PC1 is closer to the data whereas, finally, if we come to the case of $\mathrm{Ca}$, the bell-shaped trend of the radii between ${ }^{40} \mathrm{Ca}$ and ${ }^{48} \mathrm{Ca}$ is not reproduced by DFT calculations and points, most likely, to correlations that are not captured by EDFs.

\section{Examples of time-dependent calculations for vibrational states}

It is well known that the excitation spectra of atomic nuclei display an extremely rich phenomenology. The lowest nuclear excitations can be as low as $11 \mathrm{keV}$ (first excited state in ${ }^{237} \mathrm{U}$ ) or as large as $6 \mathrm{MeV}$ (first excited state in

\footnotetext{
${ }^{5}$ Only functionals with specific ratios of the neutron-proton vs. neutron-neutron spin-orbit force reproduce the kink, as explained in [34].
} 
$\left.{ }^{16} \mathrm{O}\right)$. Pairing and shell effects play obviously a role in determining the energy scales and the quantum numbers of the excited states, and yet the major role has been known for a long time to be played by the nuclear shape. If the nucleus is spherical, its low-lying spectrum is characterised by the presence of collective vibrations (originally interpreted as those of a liquid drop, and now well understood in a more microscopic fashion as we discuss below with reference to EDFs). A deformed nucleus displays instead a rotational-like spectrum. For a discussion on these topics cf., e.g., Chapter 12 of Ref. [6].

I will restrict myself here to examples of collective, high-lying vibrations known as giant resonances [77,78]. They are coherent modes, that dominate the response of nuclei to external probes like photons or light ions (e.g., $\alpha$-particles), in the energy region around $\approx 10-30 \mathrm{MeV}$. Their properties are consistent with the assumption of an elastic behaviour of the nucleus [79]. They carry different values of the spatial angular momentum $L$, spin $S$, and isospin $T$. The states in which nucleons with opposite spin vibrate in phase (out of phase) have $S=0(S=1)$ and are called electric (magnetic) resonances; if nucleons with opposite isospin, i.e. neutrons and protons, vibrate in phase (out of phase) one has $T=0(T=1)$, and speaks of isoscalar (isovector) resonances. As for $L$, the values $L=0,1,2 \ldots$ correspond to monopole, dipole, quadrupole ... Collective states do not show up for $L$ larger that 4 or 5 .

Giant resonances are, as a rule, well described by the time-dependent extension of the static DFT theory that we have discussed in Sec. 3. The extension of the HK theorem to the time-dependent case has been proposed, in the context of electronic systems, by Runge and Gross in Ref. [80] (cf. also Ref. [81]). More precisely, given a system whose behaviour is governed by an Hamiltonian

$$
H^{\prime}=H+v_{\text {pert }}(t)
$$

where the second term on the r.h.s. is a perturbing time-dependent potential, the Runge-Gross theorem establishes a one-to-one correspondence between $v_{\text {pert }}(t)$ and the time-dependent density $\rho(t)$, that is,

$$
v_{\text {pert }}(\boldsymbol{r}, t) \Leftrightarrow \rho(\boldsymbol{r}, t)
$$

For a pedagogical introduction to the subject, I advise to consult Chapter 4 of Ref. [82].

TDDFT can also be approached within the Kohn-Sham scheme. In fact, the time-dependent density can be expressed in terms of time-dependent Kohn-Sham orbitals,

$$
\rho(\boldsymbol{r}, t)=\sum_{j}\left|\phi_{j}(\boldsymbol{r}, t)\right|^{2},
$$

by generalizing Eq. (10). These orbitals must satisfy time-dependent Schrödinger equations of the type

$$
i \hbar \frac{\partial}{\partial t} \phi_{j}(\boldsymbol{r}, t)=\left[-\frac{\hbar^{2}}{2 m} \nabla^{2}+v_{\mathrm{KS}}(\boldsymbol{r}, t)+v_{\mathrm{pert}}(\boldsymbol{r}, t)\right] \phi_{j}(\boldsymbol{r}, t) .
$$

It is far from being obvious that the potential $v_{\mathrm{KS}}(\boldsymbol{r}, t)$ is the simple time-dependent extension of the one introduced in Eq. (16). This is, however, the simple approximation that is called adiabatic time-dependent DFT, and the only one that has been used so far in nuclear physics.

There are several techniques to solve the time-dependent equations (41). The direct solution can be found as follows. If $h(t)$ is the sum of the kinetic energy plus $v_{\mathrm{KS}}$, and the perturbing potential is used only to determine the initial conditions, the time evolution of the density $\rho$ is deduced from the equation of motion

$$
i \hbar \frac{d}{d t} \rho(t)=[h(t), \rho(t)] .
$$

Given an initial condition in which $\rho\left(t=t_{0}\right)$ is different from the stationary ground-state density, this latter equation can be solved by defining a time-evolution operator $\hat{U} \equiv e^{-i \frac{\Delta t}{\hbar} \cdot h}$, and by applying it to the density. Starting from the density at time $t_{0}$, one can write $\rho\left(t_{0}+\Delta t\right)=\hat{U} \rho\left(t=t_{0}\right)$, where $\Delta t$ is an appropriate time step. A useful introduction to this method can be found in Refs. [21,83].

The time-dependent equation (42) can be linearized in the case of small external perturbations. This leads to the so-called Random Phase Approximation (RPA) equations. The derivation of the RPA equations from Eq. (42) can be found, e.g., in Chapter 8 of Ref. [5]. The reader can also benefit from other standard textbooks like Ref. [84]. Moreover, a Skyrme-RPA code has been published a few years ago and the related paper [85] includes useful practical hints. The RPA equations, written on a basis or particle-hole $(p h)$ excitations on top of the ground-state, read

$$
\left(\begin{array}{cc}
A & B \\
-B & -A
\end{array}\right)\left(\begin{array}{l}
X^{(n)} \\
Y^{(n)}
\end{array}\right)=\hbar \omega_{n}\left(\begin{array}{l}
X^{(n)} \\
Y^{(n)}
\end{array}\right) \text {. }
$$

This is an eigenvalue equation, and the solutions are labelled by the index $n$. $\hbar \omega_{n}$ are the eigenvalues and, for each of them, an eigenvector which is made up with the so-called forward-going and backward-going amplitudes $X_{p h}^{(n)}$ and 
$Y_{p h}^{(n)}$ is defined ${ }^{6} . X_{p h}^{(n)}\left(Y_{p h}^{(n)}\right)$ represents the probability amplitude that a transition from the orbital $h$ to $p\left(p\right.$ to $\left.h^{7}\right)$ contributes to the $n$-th vibrational mode. The matrix elements that appear in Eq. (43) are defined by

$$
\begin{aligned}
& A_{p h, p^{\prime} h^{\prime}}=\delta_{h h^{\prime}} \delta_{p p^{\prime}}\left(\varepsilon_{p}-\varepsilon_{h}\right)+\int d^{3} r d^{3} r^{\prime} \phi_{p}^{*}(\boldsymbol{r}) \phi_{h^{\prime}}^{*}\left(\boldsymbol{r}^{\prime}\right) \frac{\delta h(\boldsymbol{r})}{\delta \rho\left(\boldsymbol{r}^{\prime}\right)} \phi_{h}(\boldsymbol{r}) \phi_{p^{\prime}}\left(\boldsymbol{r}^{\prime}\right), \\
& B_{p h, p^{\prime} h^{\prime}}=\int d^{3} r d^{3} r^{\prime} \phi_{p}^{*}(\boldsymbol{r}) \phi_{p^{\prime}}^{*}\left(\boldsymbol{r}^{\prime}\right) \frac{\delta h(\boldsymbol{r})}{\delta \rho\left(\boldsymbol{r}^{\prime}\right)} \phi_{h}(\boldsymbol{r}) \phi_{h^{\prime}}\left(\boldsymbol{r}^{\prime}\right),
\end{aligned}
$$

where $\varepsilon$ and $\phi$ are the Kohn-Sham energies and orbitals that have been introduced in Sec. 3.1, together with the Kohn-Sham Hamiltonian $h$ [cf. Eq. (16)]. The physical interpretation of $\frac{\delta h(\boldsymbol{r})}{\delta \rho\left(\boldsymbol{r}^{\prime}\right)}$ is pretty intuitive: the changes of the density affect the single-particle Hamiltonian $h$ (kinetic energy plus $v_{\mathrm{KS}}$ ) and this, in turn, produces a rearrangement of the system. In other words, the system will perform harmonic oscillations around the ground-state that are governed by the residual force

$$
V_{\text {res }}\left(\boldsymbol{r}, \boldsymbol{r}^{\prime}\right) \equiv \frac{\delta h(\boldsymbol{r})}{\delta \rho\left(\boldsymbol{r}^{\prime}\right)}
$$

We will show some illustrative RPA results in what follows.

An external electromagnetic field (photon) excites, in a practically exclusive way, the so-called isovector dipole modes. The response is in fact dominated by a resonance (Isovector Giant Dipole Resonance, IVGDR) that lies at an energy $\approx 80 A^{-1 / 3}$. One can see that in this case the photon wavelength is much larger than the nuclear size and the associated electric field, that acts on the protons, is uniform in space and consistent with a potential that is linear in the proton coordinates $\boldsymbol{r}_{p}$. It is also very easy to check that, if we take a potential of the type $e \sum_{p} \boldsymbol{r}_{p}$, and we transform it to the center-of-mass system, it becomes

$$
\hat{O}_{\text {IV dipole }}=e \frac{N}{A} \sum_{p} \boldsymbol{r}_{p}-e \frac{Z}{A} \sum_{n} \boldsymbol{r}_{n} .
$$

In the center-of-mass system, protons and neutrons are displaced in opposite directions and the strong neutron-proton interaction acts as a restoring force.

Recently, much interest has been devoted to the isovector dipole strength that lies below the IVGDR. It has been theoretically suggested that in nuclei with large neutron-proton imbalance, the excess neutrons may perform oscillations with respect to a core with equal number of neutrons and protons. Experiments have shown a non-negligible fraction of strength in some systems; whether this corresponds to the coherent vibrations of all excess neutrons or not, is still subject of debate. The name "pygmy" dipole resonance (PDR) has been widely employed to denote peaks lying below the large IVGDR peak; it should be stressed once again that the appearance of a peak does not ensure the existence of a resonance. The reader can go deeper into this topic by means of Refs. [17,86,87].

In Fig. 11 we show strength functions resulting from RPA calculations with different Skyrme functionals (SGII from Ref. [93], SkI3 from Ref. [34] and SLy5 from Ref. [51]). Strength functions are defined by

$$
S(E)=\sum_{n}|\langle n|\hat{O}| 0\rangle|^{2} \delta\left(E-\hbar \omega_{n}\right)
$$

where the RPA eigenvalues $\hbar \omega_{n}$ have been previously defined, and the corresponding eigenvectors $|n\rangle$ can be written in terms of the $X$ and $Y$ amplitudes (see Refs. $[5,84,85]$ ). $|0\rangle$ is the ground-state, and $\hat{O}$ is a generic operator. Panels (a) correspond to the choice of the isovector operator (46), and the main IVGDR peak is compared with the photoabsorbtion result of Ref. [88], while the low-lying peak which can be referred to as PDR (with the aforementioned words of caution) is compared with the experimental findings of Refs. [89,90]. Panels (b) show instead the response to the isoscalar dipole operator,

$$
\hat{O}_{\text {IS dipole }}=\sum_{i}\left(r_{i}^{3}-\frac{3}{5}\left\langle r^{2}\right\rangle r_{i}\right) Y_{1 M}\left(\hat{r}_{i}\right) .
$$

Details for this choice can be found in Ref. [92] (cf. also Ref. [94]). The result for the main resonance excited by this operator can be compared with the result of experiments like inelastic $\left(\alpha, \alpha^{\prime}\right)$ scattering, as we have done by taking the finding of [91]. The results in the figure seem to indicate that the low-lying dipole response has more isoscalar than isovector character.

\footnotetext{
${ }^{6}$ Here, and in what follows, $h, h^{\prime}$ are used to label orbitals below the Fermi energy and $p, p^{\prime}$ are used to label orbitals above the Fermi energy.

7 Note that introducing the backward-going amplitudes is consistent with the linearity of the response, but implies relaxing the assumption that the ground-state is made up with the auxiliary orbitals filled up to the Fermi energy, and empty above this energy.
} 

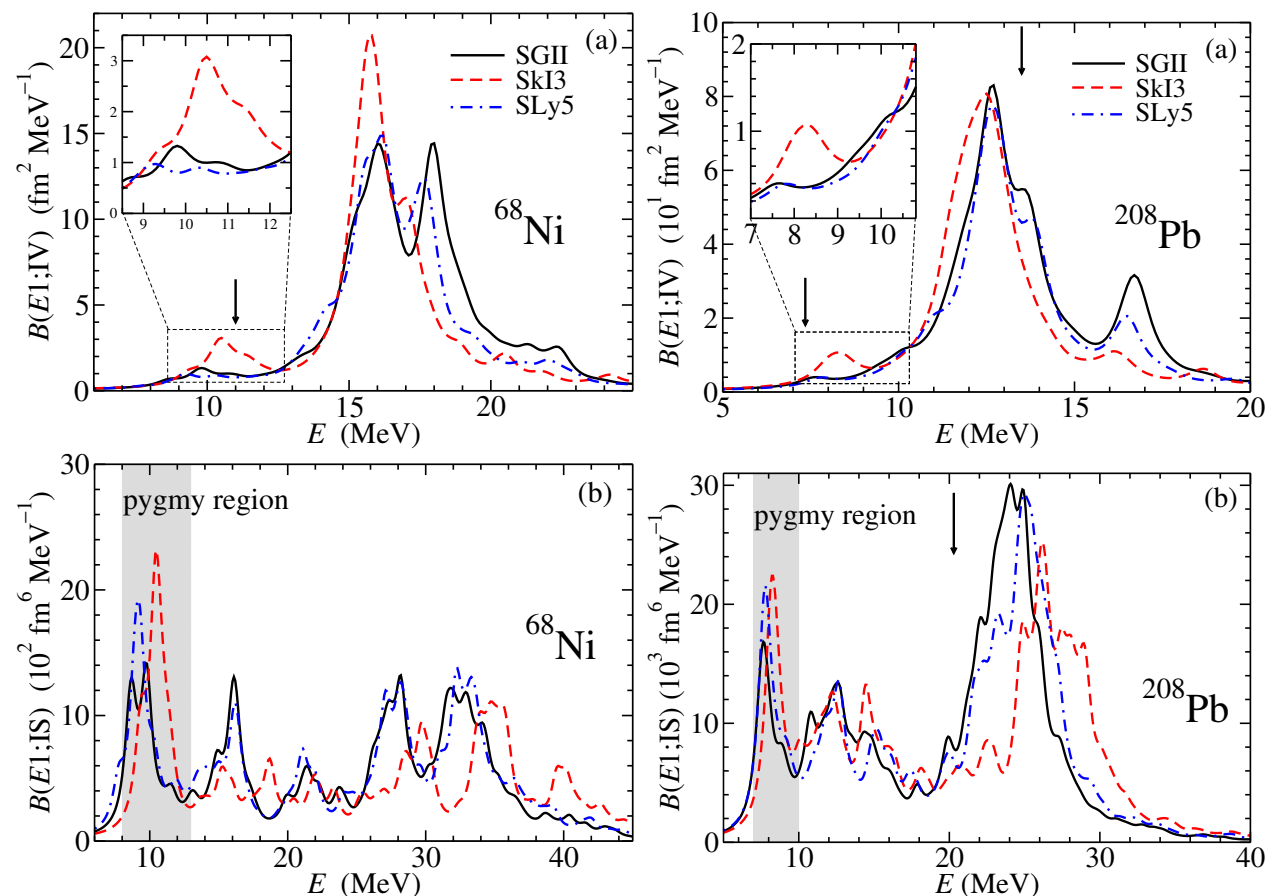

Fig. 11. Strength functions corresponding to the isovector (a) and isoscalar (b) dipole response of ${ }^{68} \mathrm{Ni}\left(\right.$ left panels) and ${ }^{208} \mathrm{~Pb}$ (right panels) as a function of the excitation energy. The insets display on a larger scale the low-energy, or pygmy, region. The predictions obtained with different Skyrme functionals are depicted (cf. the main text). The results are averaged with Lorentzian functions having $1 \mathrm{MeV}$ width. Black arrows indicate the experimental results from Refs. [88-91]. Figure taken from Ref. [92].

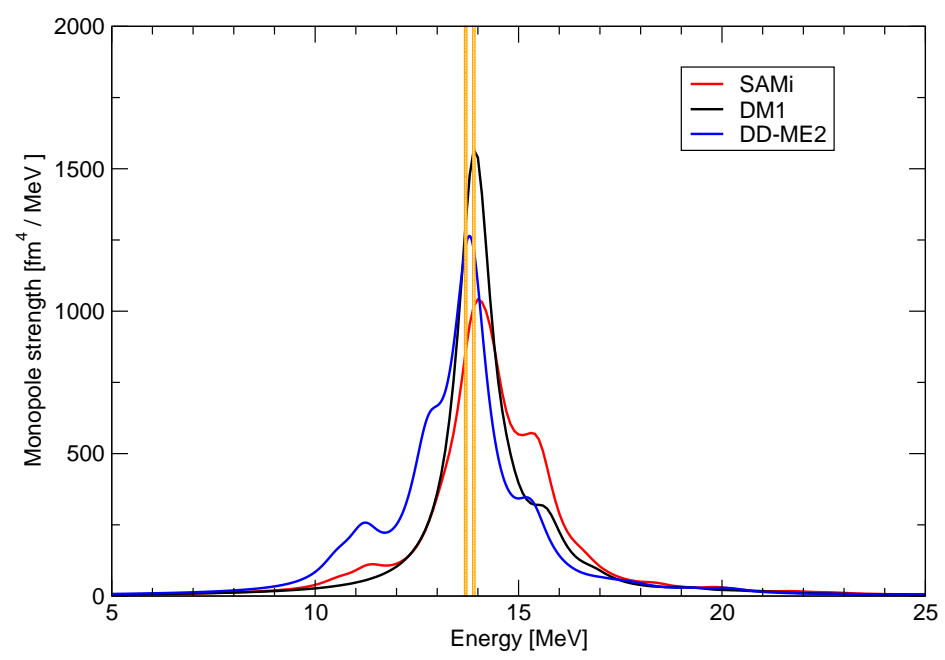

Fig. 12. RPA calculations of the monopole strength in ${ }^{208} \mathrm{~Pb}$, performed by using the nonrelativistic Skyrme-type functional SAMi [92], the Gogny-type functional DM1 [95], and the relativistic functional DDME2 [96]. The vertical lines show the peak energy obtained in the experiments performed at TAMU (13.9 MeV) and RCNP (13.7 MeV).

In fact, $\left(\alpha, \alpha^{\prime}\right)$ scattering excites only isoscalar electric giant resonances, in keeping with the rather pure $S=0, T=0$ character of the ground-state of ${ }^{4} \mathrm{He}$. This probe has been use extensively starting from the 1980 s to identify the Giant Monopole Resonance (GMR) and the Giant Quadrupole Resonance (GQR) [77,78]. The GMR is of interest because its excitation energy is related, albeit not straighfowardly, to the nuclear incompressibility $K_{\infty}$ (cf. [94] and references therein) which is a key parameter of the nuclear equation of state. Obviously, the equation of state and in particular 
$K_{\infty}$ are not direct observables. Yet, they have applications in astrophysics and in the physics of heavy-ion collisions. To mention only one of these applications, the equation of state with its related value of $K_{\infty}$ is an input for simulations of the collapse that some massive stars undergo at the end of their life, before the supernova explosion (cf., e.g., Sec. 3.3 of Ref. [97]).

The incompressibility of symmetric nuclear matter is related to the saturation properties that we have discussed in Secs. 2.3 and 4.1. As we said, the energy per particle in symmetric nuclear matter has a minimum $E / A$ at $\rho_{0}=$ $0.16 \mathrm{fm}^{-3}$. Around this minimum, one can write

$$
\frac{E}{A}(\rho)=\frac{E}{A}\left(\rho_{0}\right)+\frac{1}{2} K_{\infty}\left(\frac{\rho-\rho_{0}}{\rho_{0}}\right)^{2}+\ldots,
$$

where the factors come from the fact that this expansion has been conceived and written in terms of the Fermi momentum, $k_{F}$, and where the compression modulus or nuclear incompressibility $K_{\infty}$ shows up as

$$
K_{\infty}=9 \rho_{0}^{2} \frac{d^{2}}{d \rho^{2}}\left(\frac{E}{A}\right)_{\rho=\rho_{0}} .
$$

This quantity is related with the compressibility $\chi$ that has one knows from elementary physics, $\chi \equiv-\frac{1}{V}\left(\frac{\partial P}{\partial V}\right)^{-1}$ where $V$ and $P$ are volume and pressure, respectively, by

$$
K_{\infty}=\frac{9}{\rho_{0}} \chi^{-1}
$$

There is a relationship between the incompressibility of nuclear matter and the compression properties of finite nuclei. In particular, DFT calculations shows a nice correlation between the value of $K_{\infty}$ associated with a given functional, and the corresponding GMR energies extracted from RPA. In Fig. 12 we show the strength functions from RPA calculations performed with different functionals, or Skyrme, Gogny and covariant type. They are characterised by values of $K_{\infty}$ equal to $225 \mathrm{MeV}$ (D1M), $230 \mathrm{MeV}$ (SAMi) and $251 \mathrm{MeV}$ (DD-ME2). As these three models all reproduce the experimental findings in ${ }^{208} \mathrm{~Pb}$, one can say that a value of $K_{\infty}$ around the mentioned ones is the correct value. Measurements in open-shell, or deformed nuclei, may point to slightly different values as discussed at length in Ref. [94].

\section{Conclusions}

DFT for atomic nuclei is a lively field, in which active research is carried out right now in close connection with the new experimental findings. The scope of this paper is not at all giving a complete account of the achievements in this domain, but simply to introduce a new student to the topic, and let her or him continue by moving to the specialized literature.

After staring from a review of some basic nuclear phenomenology, I have introduced the basics of DFT. The hope is to have been able to convince readers that this theory has several advantages. Its basic principle is very intuitive: one exploits the fact that the total energy can be written as a functional of the density only, and simply chooses a way to minimize the energy. In pirnciple, all quantities of interest can be derived from this minimization (radii, deformations, multipole moments etc.). The main advantage of DFT is its broad range of applicability. The main shortcoming is that there is not a unique way to build a functional, and not a clear path to systematically improve the functionals in use.

I have dicusssed some examples of nuclear EDFs with applications to calculations of masses, radii, nuclear superfluidity and giant resonances. There is a long list of very interesting and relevant subjects that could not fit the format of the lecture and of the paper, or that I have skipped for other reasons. Among them, I can quote symmetry breaking and restoration, multi-reference DFT, applications to low-lying spectroscopy, rotations and high-spin phenomena, super-heavy elements, charge-exchange transitions (the list is of course not exhaustive). I have also not discussed two topics that are very instrumental for the development of this field, namely EDFs from underlying theories, and confrontations between DFT and many-body theory.

Finally, I hope that readers can just find this paper motivating enough so that they wish to spend more time in understanding how DFT can lead to a unified picture of the nuclear landscape.

\section{Acknowledgments}

This work is, among the rest, also the result of many fruitful collaborations during the last decades. I would like to thank, for the common work and the beneficial discussions on the topics that are dealt with in this paper, K. 
Bennaceur, P.F. Bortignon, J. Dobaczewski, T. Duguet, M. Grasso, E. Khan, D. Lacroix, H. Liang, J. Margueron, J. Meng, J. Meyer, Y. Niu, N. Paar, P.-G. Reinhard, P. Ring, X. Roca-Maza, H. Sagawa, N. Van Giai, E. Vigezzi, and D. Vretenar. Funding from the European Union's Horizon 2020 research and innovation programme under grant agreement No 654002 is also acknowledged.

\section{Appendix}

The total energy of a many-body system, calculated over a Slater determinant, has the general form

$$
E=\sum_{j}\langle j|\hat{O}(1)| j\rangle+\frac{1}{2} \sum_{i j}\langle i j|\hat{O}(2)| i j\rangle+\frac{1}{3 !} \sum_{i j l}\langle i j l|\hat{O}(3)| i j l\rangle+\ldots
$$

where $O(n)$ is a $n$-body operator, and the matrix elements must be antisimmetrized. The kinetic energy is the only one-body operator and its evaluation brings us back to Eq. (11). The two-body interaction gives

$$
V=\frac{1}{2} \sum_{i j}\langle i j| V\left(1-P_{12}|i j\rangle=\frac{1}{2} \sum_{i j} \int d^{3} r d^{3} r^{\prime} \phi_{i}^{*}(\boldsymbol{r}) \phi_{j}^{*}\left(\boldsymbol{r}^{\prime}\right) V\left(\boldsymbol{r}, \boldsymbol{r}^{\prime}\right)\left(1-P_{12}\right) \phi_{i}(\boldsymbol{r}) \phi_{j}\left(\boldsymbol{r}^{\prime}\right),\right.
$$

where $P_{12}$ is the operator that exchanges particles 1 and 2 , and produces the antisimmetrization of the matrix elements.

We now introduce the simplified Skyrme force (19) and we use the abbreviation $g(\boldsymbol{r})=t_{0}+\frac{1}{6} t_{3} \rho^{\alpha}$. The operator $P_{12}$ is equal to $P_{M} P_{\sigma} P_{\tau}$, that exchanges position, spin and isospin of the two particles, respectively. As we deal with a $\delta$-force, $P_{M}$ can be replaced by 1 . The potential energy (53) becomes

$$
V=\frac{1}{2} \sum_{i j} \int d^{3} r \phi_{i}^{*}(\boldsymbol{r}) \phi_{j}^{*}(\boldsymbol{r}) g(\boldsymbol{r})\left(1-P_{\sigma} P_{\tau}\right) \phi_{i}(\boldsymbol{r}) \phi_{j}(\boldsymbol{r}) .
$$

As it has been defined in the main text, $P_{\sigma}=\frac{1+\boldsymbol{\sigma}_{1} \cdot \boldsymbol{\sigma}_{2}}{2}$ and $P_{\tau}=\frac{1+\boldsymbol{\tau}_{1} \cdot \boldsymbol{\tau}_{2}}{2}$. Using elementary properties of the Pauli matrices, one can show that the expectation value of $\boldsymbol{\sigma}_{1} \cdot \boldsymbol{\sigma}_{2}$ vanishes on a Slater determinant made up with an even number of particles that needs to respect time-reversal invariance. Thus, $P_{\sigma}$ can be replaced by $1 / 2$. If we do not allow proton-neutron mixing, that is, we assume that the two isospin states remain distinguishable, $P_{\tau}$ gives a vanishing result if $i$ and $j$ correspond to particles of a different kind and 1 if the two particles $i$ and $j$ are both neutrons or both protons. Thus, we arrive at

$$
V=\frac{1}{2} \sum_{i j} \int d^{3} r \phi_{i}^{*}(\boldsymbol{r}) \phi_{j}^{*}(\boldsymbol{r}) g(\boldsymbol{r})\left(1-\frac{1}{2} \delta\left(q_{i}, q_{j}\right)\right) \phi_{i}(\boldsymbol{r}) \phi_{j}(\boldsymbol{r}),
$$

where $q_{i}$ is the charge of the particle in the orbital $i$. The sums over $i$ and $j$ can now be performed to obtain the density.

The result reads

$$
\begin{aligned}
& E\left[\rho_{n}, \rho_{p}\right]=\int d^{3} r \mathcal{E}\left[\rho_{n}, \rho_{p}\right] \\
& \mathcal{E}\left[\rho_{n}, \rho_{p}\right]=\frac{\hbar^{2}}{2 m} \tau+\frac{1}{2} t_{0}\left[\rho^{2}-\frac{1}{2}\left(\rho_{n}^{2}+\rho_{p}^{2}\right)\right]+\frac{1}{12} t_{3}\left[\rho^{\alpha+2}-\frac{1}{2} \rho^{\alpha}\left(\rho_{n}^{2}+\rho_{p}^{2}\right)\right]
\end{aligned}
$$

that is, we have shown the validity of Eq. (20).

\section{References}

1. A.L. Fetter, J.D. Walecka, Quantum Theory of Many-Particle Systems (McGraw-Hill, 1971)

2. R. Machleidt, D. Entem, Physics Reports 503, 1 (2011)

3. E. Epelbaum, U.G. Meißner, Annual Review of Nuclear and Particle Science 62, 159 (2012)

4. K. Hebeler, J. Holt, J. Menendez, A. Schwenk, Annual Review of Nuclear and Particle Science 65, 457 (2015)

5. P. Ring, P. Schuck, The Nuclear Many-Body Problem (Springer, 1980)

6. K. Heyde, Basic Ideas and Concepts in Nuclear Physics (IoP Publishing, 1994)

7. J. Suhonen, From Nucleons to Nucleus. Concepts of Microscopic Nuclear Theory. (Springer, 2007)

8. C. Bertulani, Nuclear Physics in a Nutshell (Princeton University Press, 2007) 
9. M. Bender, P.H. Heenen, P.G. Reinhard, Rev. Mod. Phys. 75, 121 (2003)

10. G. Lalazissis, P. Ring, D. Vretenar, eds., Extended Density Functionals in Nuclear Structure Physics. Lecture Notes in Physics. Vol. 641 (Springer, 2004)

11. T. Duguet, Lecture Notes in Physics. Vol. 879 (Springer, 2004), p. 293

12. D. Vretenar, A. Afanasjev, G. Lalazissis, P. Ring, Physics Reports 409, 101 (2005)

13. T. Nikšić, D. Vretenar, P. Ring, Progress in Particle and Nuclear Physics 66, 519 (2011)

14. J. Dobaczewski, Interactions, symmetry breaking, and effective fields from quarks to nuclei. (a primer in nuclear theory) (2003), arXiv:nucl-th/0301069

15. G. Colò, Density functional theory (dft) for atomic nuclei: a simple introduction (2018), arXiv:nucl-th/1807.02643

16. O. Sorlin, M.G. Porquet, Progress in Particle and Nuclear Physics 61, 602 (2008)

17. N. Paar, D. Vretenar, E. Khan, G. Colò, Reports on Progress in Physics 70, 691 (2007)

18. A. Koning, J. Delaroche, Nuclear Physics A 713, 231 (2003)

19. W. Huang, G. Audi, M. Wang, F. Kondev, S. Naimi, X. Xu, Chinese Physics C 41, 030002 (2017)

20. M. Wang, G. Audi, F. Kondev, W. Huang, S. Naimi, X. Xu, Chinese Physics C 41, 030003 (2017)

21. T. Nakatsukasa, K. Matsuyanagi, M. Matsuo, K. Yabana, Rev. Mod. Phys. 88, 045004 (2016)

22. D. Davesne, J. Navarro, J. Meyer, K. Bennaceur, A. Pastore, Phys. Rev. C 97, 044304 (2018)

23. P. Hohenberg, W. Kohn, Phys. Rev. 136, B864 (1964)

24. R. Dreizler, E. Gross, Density Functional Theory (Springer, 1990)

25. J. Margueron, R. Hoffmann Casali, F. Gulminelli, Phys. Rev. C 97, 025805 (2018)

26. J.P. Perdew, K. Schmidt, AIP Conference Proceedings 577, 1 (2001)

27. W. Kohn, L.J. Sham, Phys. Rev. 140, A1133 (1965)

28. R.A. Broglia, G. Colò, G. Onida, H.E. Roman, Solid State Physics of Finite Systems (Springer, 2004)

29. J. Drut, R. Furnstahl, L. Platter, Progress in Particle and Nuclear Physics 64, 120 (2010)

30. T.H.R. Skyrme, The Philosophical Magazine: A Journal of Theoretical Experimental and Applied Physics 1, 1043 (1956)

31. T. Skyrme, Nuclear Physics 9, 615 (1958)

32. D. Vautherin, D.M. Brink, Phys. Rev. C 5, 626 (1972)

33. Y. Engel, D. Brink, K. Goeke, S. Krieger, D. Vautherin, Nuclear Physics A 249, 215 (1975)

34. P.G. Reinhard, H. Flocard, Nuclear Physics A 584, 467 (1995)

35. M. Kortelainen, J. McDonnell, W. Nazarewicz, E. Olsen, P.G. Reinhard, J. Sarich, N. Schunck, S.M. Wild, D. Davesne, J. Erler et al., Phys. Rev. C 89, 054314 (2014)

36. J. Dobaczewski, J. Dudek, Acta Physica Polonica B 27, 45 (1996)

37. F. Raimondi, B.G. Carlsson, J. Dobaczewski, Phys. Rev. C 83, 054311 (2011)

38. D. Davesne, P. Becker, A. Pastore, J. Navarro, Annals of Physics 375, 288 (2016)

39. D.G. Ireland, W. Nazarewicz, Journal of Physics G: Nuclear and Particle Physics 42, 030301 (2015)

40. S. Fayans, E. Trykov, D. Zawischa, Nuclear Physics A 568, 523 (1994)

41. S.A. Fayans, Journal of Experimental and Theoretical Physics Letters 68, 169 (1998)

42. J. Dechargé, D. Gogny, Phys. Rev. C 21, 1568 (1980)

43. J. Berger, M. Girod, D. Gogny, Computer Physics Communications 63, 365 (1991)

44. H. Nakada, Phys. Rev. C 78, 054301 (2008)

45. X. Roca-Maza, N. Paar, Progress in Particle and Nuclear Physics 101, 96 (2018)

46. D.M. Brink, R.A. Broglia, Nuclear Superfluidity: Pairing in Finite Systems (Cambridge University Press, 2005)

47. A. Bohr, B.R. Mottelson, Nuclear Structure, Vol. II (Benjamin, New York, 1975)

48. W. Satuła, J. Dobaczewski, W. Nazarewicz, Phys. Rev. Lett. 81, 3599 (1998)

49. T. Duguet, P. Bonche, P.H. Heenen, J. Meyer, Phys. Rev. C 65, 014311 (2001)

50. A. Bohr, B.R. Mottelson, D. Pines, Phys. Rev. 110, 936 (1958)

51. E. Chabanat, P. Bonche, P. Haensel, J. Meyer, R. Schaeffer, Nuclear Physics A 635, 231 (1998)

52. J. Li, G. Colò, J. Meng, Phys. Rev. C 78, 064304 (2008)

53. A. Bulgac, Hartree-fock-bogoliubov approximation for finite systems (2000), arXiv:nucl-th/9907088

54. J. Dobaczewski, W. Nazarewicz, T.R. Werner, J.F. Berger, C.R. Chinn, J. Dechargé, Phys. Rev. C 53 (1996)

55. G. Bertsch, H. Esbensen, Annals of Physics 209, 327 (1991)

56. M. Yamagami, J. Margueron, H. Sagawa, K. Hagino, Phys. Rev. C 86, 034333 (2012)

57. E. Perlińska, S.G. Rohoziński, J. Dobaczewski, W. Nazarewicz, Phys. Rev. C 69, 014316 (2004)

58. M. Lacombe, B. Loiseau, J.M. Richard, R.V. Mau, J. Côté, P. Pirès, R. de Tourreil, Phys. Rev. C 21, 861 (1980)

59. E. Garrido, P. Sarriguren, E. Moya de Guerra, U. Lombardo, P. Schuck, H.J. Schulze, Phys. Rev. C 63, 037304 (2001)

60. F. Barranco, P.F. Bortignon, R.A. Broglia, G. Colò, P. Schuck, E. Vigezzi, X. Viñas, Phys. Rev. C 72, 054314 (2005)

61. H. Esbensen, G. Bertsch, Nuclear Physics A 542, 310 (1992)

62. K. Hagino, H. Sagawa, J. Carbonell, P. Schuck, Phys. Rev. Lett. 99, 022506 (2007)

63. N. Pillet, N. Sandulescu, P. Schuck, J.F. Berger, Phys. Rev. C 81, 034307 (2010)

64. A. Sedrakian, J.W. Clark, Superfluidity in nuclear systema and neutron stars (2018), arXiv:nucl-th/1802.00017

65. H. Sagawa, C.L. Bai, G. Colò, Physica Scripta 91, 083011 (2016)

66. S. Goriely, N. Chamel, J.M. Pearson, Phys. Rev. C 88, 024308 (2013)

67. M. Kortelainen, T. Lesinski, J. Moré, W. Nazarewicz, J. Sarich, N. Schunck, M.V. Stoitsov, S. Wild, Phys. Rev. C 82, $024313(2010)$ 
68. M. Kortelainen, J. McDonnell, W. Nazarewicz, P.G. Reinhard, J. Sarich, N. Schunck, M.V. Stoitsov, S.M. Wild, Phys. Rev. C 85, 024304 (2012)

69. T. Nikšić, D. Vretenar, P. Ring, Phys. Rev. C 78, 034318 (2008)

70. P. Mller, A. Sierk, T. Ichikawa, H. Sagawa, Atomic Data and Nuclear Data Tables 109-110, 1 (2016)

71. M. Liu, N. Wang, Y. Deng, X. Wu, Phys. Rev. C 84, 014333 (2011)

72. A. Afanasjev, S. Agbemava, D. Ray, P. Ring, Physics Letters B 726, 680 (2013)

73. J. Erler, N. Birge, M. Kortelainen, W. Nazarewicz, E. Olsen, A.M. Perhac, M. Stoitsov, Nature 486, 509 (2012)

74. P. Moller, J. Nix, W. Myers, W. Swiatecki, Atomic Data and Nuclear Data Tables 59, 185 (1995)

75. J.P. Delaroche, M. Girod, J. Libert, H. Goutte, S. Hilaire, S. Péru, N. Pillet, G.F. Bertsch, Phys. Rev. C 81, 014303 (2010)

76. I. Angeli, K. Marinova, Atomic Data and Nuclear Data Tables 99, 69 (2013)

77. P.F. Bortignon, A. Bracco, R.A. Broglia, Giant Resonances: Nuclear Structure at Finite Temperature (Harwood, 1999)

78. M.N. Harakeh, A. van der Woude, Giant Resonances Fundamental High-Frequency Modes of Nuclear Excitation (Oxford University Press, New York, 2001)

79. G. Bertsch, Annals of Physics 86, 138 (1974)

80. E. Runge, E.K.U. Gross, Phys. Rev. Lett. 52, 997 (1984)

81. R. van Leeuwen, Phys. Rev. Lett. 80, 1280 (1998)

82. C. Fiolhais, F. Nogueira, M. Marques, eds., A primer in Density Functional Theory. Lecture Notes in Physics. Vol. 620 (Springer, 2003)

83. P. Reinhard, E. Suraud, Introduction to Cluster Dynamics (Wiley, 2004)

84. D. Rowe, Nuclear Collective Motion (Methuen, London, 1970)

85. G. Colò, L. Cao, N.V. Giai, L. Capelli, Computer Physics Communications 184, 142 (2013)

86. D. Savran, T. Aumann, A. Zilges, Progress in Particle and Nuclear Physics 70, 210 (2013)

87. A. Bracco, F.C.L. Crespi, E.G. Lanza, The European Physical Journal A 51, 99 (2015)

88. B.L. Berman, S.C. Fultz, Rev. Mod. Phys. 47, 713 (1975)

89. O. Wieland, A. Bracco, F. Camera, G. Benzoni, N. Blasi, S. Brambilla, F.C.L. Crespi, S. Leoni, B. Million, R. Nicolini et al., Phys. Rev. Lett. 102, 092502 (2009)

90. N. Ryezayeva, T. Hartmann, Y. Kalmykov, H. Lenske, P. von Neumann-Cosel, V.Y. Ponomarev, A. Richter, A. Shevchenko, S. Volz, J. Wambach, Phys. Rev. Lett. 89, 272502 (2002)

91. U. Garg, Nuclear Physics A 649, 66 (1999)

92. X. Roca-Maza, G. Pozzi, M. Brenna, K. Mizuyama, G. Colò, Phys. Rev. C 85, 024601 (2012)

93. N.V. Giai, H. Sagawa, Physics Letters B 106, 379 (1981)

94. U. Garg, G. Colò, Progress in Particle and Nuclear Physics 101, 55 (2018)

95. S. Goriely, S. Hilaire, M. Girod, S. Péru, Phys. Rev. Lett. 102, 242501 (2009)

96. G.A. Lalazissis, T. Nikšić, D. Vretenar, P. Ring, Phys. Rev. C 71, 024312 (2005)

97. H.T. Janka, Annual Review of Nuclear and Particle Science 62, 407 (2012) 